\title{
MLL-AF9- and HOXA9-mediated acute myeloid leukemia stem cell self-renewal requires JMJD1C
}

\author{
Nan Zhu, ${ }^{1,2}$ Mo Chen, ${ }^{3}$ Rowena Eng, ${ }^{1}$ Joshua Dejong, ${ }^{2}$ Amit U. Sinha, ${ }^{1}$ Noushin F. Rahnamay, ${ }^{1}$ Richard Koche, ${ }^{1}$ \\ Fatima Al-Shahrour, ${ }^{4}$ Janna C. Minehart, ${ }^{1}$ Chun-Wei Chen, ${ }^{1}$ Aniruddha J. Deshpande, ${ }^{1}$ Haiming Xu, ${ }^{1}$ S. Haihua Chu, ${ }^{1}$ \\ Benjamin L. Ebert, ${ }^{4}$ Robert G. Roeder, ${ }^{3}$ and Scott A. Armstrong ${ }^{1}$ \\ 'Cancer Biology and Genetics Program, Memorial Sloan Kettering Cancer Center, New York, New York, USA. ${ }^{2}$ Blood Research Institute, Blood Center of Wisconsin, Milwaukee, Wisconsin, USA. ${ }^{3}$ Laboratory \\ of Biochemistry and Molecular Biology, The Rockefeller University, New York, New York, USA. ${ }^{4}$ Division of Hematology, Department of Medicine, Brigham and Women's Hospital, Harvard Medical School, \\ Boston, Massachusetts, USA.
}

\begin{abstract}
Self-renewal is a hallmark of both hematopoietic stem cells (HSCs) and leukemia stem cells (LSCs); therefore, the identification of mechanisms that are required for LSC, but not HSC, function could provide therapeutic opportunities that are more effective and less toxic than current treatments. Here, we employed an in vivo shRNA screen and identified jumonji domain-containing protein JMJD1C as an important driver of MLL-AF9 leukemia. Using a conditional mouse model, we showed that loss of JMJD1C substantially decreased LSC frequency and caused differentiation of MLL-AF9- and homeobox A9-driven (HOXA9-driven) leukemias. We determined that JMJD1C directly interacts with HOXA9 and modulates a HOXA9-controlled gene-expression program. In contrast, loss of JMJD1C led to only minor defects in blood homeostasis and modest effects on HSC self-renewal. Together, these data establish JMJD1C as an important mediator of MLL-AF9- and HOXA9-driven LSC function that is largely dispensable for HSC function.
\end{abstract}

\section{Introduction}

Hematopoiesis is organized in a hierarchy in which the hematopoietic stem cell (HSC) is at the apex. HSCs have the unique ability to self-renew and differentiate into all blood lineages (1). The ability to self-renew is also a hallmark of leukemia stem cells (LSCs), but multiple studies suggest that LSC self-renewal is dependent on pathways different from those that are critical for HSC self-renewal (2-6). Understanding the different dependencies of LSC versus HSC may lead to the development of more effective, less toxic therapies.

The mixed lineage leukemia $(M L L)$ gene, the mammalian homolog of Drosophila trithorax, encodes a histone methyltransferase $(7,8)$ that is involved in regulation of homeotic genes during embryonic development (9). $M L L$ has also been shown to be required for the development and maintenance of hematopoiesis (10-12). Translocations involving $M L L$ are found in over $70 \%$ of infant leukemias and $5 \%$ to $10 \%$ of leukemias in adults and are often associated with poor prognosis (13). In $M L L$-rearranged leukemias, the N-terminal of MLL is fused to over 50 fusion partners. $M L L$ translocations show lineage specificity, and the most common translocation in acute myeloid leukemia (AML) is t $(9 ; 11)$ which encodes the oncogenic MLL-AF9 fusion protein (13). MLL-AF9 leukemia has been shown to follow an LSC model in which LSCs are enriched in a subset of leukemia cells with a specific immune phenotype $(14,15)$. Transformation by MLL-AF9 induces aberrant expression of a self-renewal-associated gene-expression program,

Conflict of interest: The authors have declared that no conflict of interest exists. Submitted: June 1, 2015; Accepted: December 18, 2015.

Reference information: / Clin Invest. 2016;126(3):997-1011. doi:10.1172/JCI82978. which includes the canonical MLL-AF9 target genes homeobox A9 (HOXA9) and Meis homeobox 1 (MEIS1) (16-21). Leukemia maintenance is dependent on the continuous presence of the fusion oncogene (22), highlighting the importance of MLL-AF9 target genes in leukemogenesis and validating them as additional entry points for therapeutic intervention.

Epigenetic regulators have been shown to play an important role in regulating self-renewal in HSC and LSC. BMI1, a component of the polycomb complex 1 (PRC1), is required for the selfrenewal of HSC and LSC in Hoxa9/Meis1-driven leukemias (23, 24). Several epigenetic regulators that are essential for MLL-AF9 leukemia, such as DOT1L and EED, are also required for HSC function (25-29). Another epigenetic regulator, CBX8, a polycomb group protein, is required for MLL-AF9 leukemia, but not HSC function (30). Loss of CBX8 impaired MLL-AF9 leukemogenesis, but had no measurable effect on normal hematopoiesis, providing an example of an epigenetic regulator that has a more prominent role in leukemia than normal HSC function.

JMJD1C is a jumonji domain-containing protein and a member of the lysine demethylase 3 (KDM3) family. First identified in a yeast 2-hybrid assay as thyroid receptor-interacting protein (TRIP8) (31), JMJD1C has been shown to be a coactivator for the androgen receptor (32). JMJD1C is required in male gametogenesis (33) and has been shown to represses neural differentiation of human embryonic stem cells by maintaining miR-302 expression (34). Germline variants of JMJD1C are associated with increased risk of developing intracranial germ cell tumors (35), and recently, shRNA approaches demonstrated a requirement for JMJD1C in leukemia maintenance (36). These results point to a possible role for JMJD1C in AML stem cell function and perhaps hematopoiesis more broadly. 
In this study, we used in vivo shRNA screening to identify MLL-AF9 targets that are essential for leukemogenesis. We characterized the role of JMJD1C, a downstream target of MLL-AF9 in leukemia and normal HSC function, using conditional loss-of-function models and found that JMJD1C is differentially required for self-renewal of LSC versus HSC. We identified the HOXA9-driven gene-expression program as a critical component of JMJD1C function in leukemogenesis and showed that HOXA9driven LSC are dependent upon JMJD1C. Our results provide an example of an epigenetic regulator that is differentially required for LSC and HSC and provide a potential therapeutic opportunity in the approximately $50 \%$ of AML cases that aberrantly express high levels of HOXA9.

\section{Results}

In vivo shRNA screen of MLL-AF9 target genes. We previously identified a list of MLL-AF9 target genes in LSC by ChIP sequencing (ChIP-seq) (28). To assess the importance of these genes in MLLAF9 leukemogenesis and to identify potential therapeutic targets, we conducted a pooled shRNA screen. To this end, we transduced tertiary MLL-AF9 leukemias with a custom lentivirus library, expressing a total of 752 shRNAs, including 737 against 149 MLLAF9 target genes, 15 against 3 positive control genes, catenin $\beta 1$ (Ctnnb1), Hoxa9, and Meis1 (Hoxa9 and Meis1 are also MLL-AF9 target genes), and 8 against negative control genes $L a c Z, R F P$, and luciferase (Supplemental Table 1; supplemental material available online with this article; doi:10.1172/JCI82978DS1). Twenty-four hours after transduction, we saved aliquots as early time-point samples and transplanted the rest into sublethally irradiated recipient mice. When mice became moribund at 2 weeks, we sacrificed them and harvested BM and spleen (SPL) samples. Using highthroughput sequencing of PCR-amplified shRNA sequence from genomic DNA, we quantified relative abundance of each hairpin shortly after transduction of shRNA and in BM and SPL cells from moribund animals (Figure 1A and Supplemental Table 1). Positive control hairpins were selected against, whereas negative control hairpins were neither selected for nor against (Figure 1B). On average, we obtained 13 million reads per sample and about 17,000× coverage for each of the shRNA hairpins (Supplemental Figure 1A). We had high reproducibility between 8 replicates, as evidenced by the tight clustering of early time points, BM, and SPL samples analyzed by unsupervised clustering (Supplemental Figure 1B). There was also tight correlation between relative abundance of each hairpin between BM and SPL samples (Supplemental Figure $1 \mathrm{C}, R^{2}=0.89$ ), suggesting that this is a high-quality screen. Consistent with the reported requirement of Ctnnb1, Hoxa9, and Meis1 in MLL-AF9 leukemogenesis $(2,37,38), 3 / 5,4 / 5$, and 3/5 hairpins, respectively, were depleted by more than 10-fold (Figure 1C). In addition, since the expression of MLL-AF9 itself is driven off a retroviral promoter by the bicistronic MSCV-IRES-GFP, we observed a greater than 10 -fold depletion of 2/2 hairpins against GFP. This is in accordance with the continued requirement of MLL-AF9 oncogene in the maintenance of MLL-AF9 leukemia (22).

We defined a positive hit in the screen by a criteria of greater than 10-fold median depletion in the BM of at least 2 hairpins against an individual gene. By this criteria, a total of 88 out of 149 genes we screened for were considered hits from the screen
(Supplemental Table 2). Next, we assessed gene-expression differences between MLL-AF9 LSC (L-GMP) and normal GMP for previously defined direct MLL-AF9 target genes $(14,28)$. We found Meis1, Jmjd1c, and Hoxa9 as the top differentially expressed MLL-AF9 target genes in mouse LSC that also were positive hits in the screen (Figure 1D). Furthermore, JMJD1C was 1 of the top 3 (top 2 are HOXA9 and A10) differentially expressed MLL-AF9 target genes (28) in a data set of human MLL and non-MLL rearranged AML (ref. 39 and Supplemental Figure 1D). Also, there was a moderate positive correlation between JMJD1C and HOXA9 expression in the AML data set analyzed $(r>0.3$, Pearson correlation, Supplemental Figure 1D). Based on these data, we decided to further assess the role of JMJD1C in leukemia and hematopoiesis.

JMJD1C is required for MLL-AF9-mediated leukemogenesis. JMJD1C has previously been reported as an H3K9me2/1 demethylase (40). To understand its role in MLL-AF9 leukemogenesis, we obtained mice with a targeted trap allele of Jmjd1c (Jmjd1 $c^{\text {tmla/+ }}$ ) where exons 8 and 9 of Jmjd1c are flanked by LoxP sites, from the European Mouse Mutant Archive (41). These mice were bred with ACTB-FLPe mice, which express the Flp recombinase, to remove the gene trap cassette flanked by flippase recognition target (FRT) sites (Figure 2A). The resulting Jmjd1 $c^{f /+}$ mice were bred to generate $J m j d 1 c^{f / f}$ mice. We transformed $\mathrm{Lin}^{-} \mathrm{Sca}^{+}{ }^{+} \mathrm{c}-\mathrm{Kit}^{+}$(LSK) cells from Jmjd1 $c^{f / f}$ and WT control mice with MLL-AF9 GFP. The transformed cells were transduced with either Cre MSCV-IRES-YFP (CRE-YFP) or empty vector control virus (MIT) and sorted for GFP/YFP-positive cells. Loss of JMJD1C greatly reduced the number of colonies in methylcellulose after 1 week in colony-forming cell (CFC) assays (Supplemental Figure 2A). In addition, we observed that reduced colony size and Wright-Giemsa staining of cells from these colonies was consistent with terminal differentiation of JMJD1C-deficient cells showing cells that resemble neutrophils and macrophages (Supplemental Figure 2B).

We next assessed the loss of JMJD1C in fully developed leukemia. To this end, we injected MLL-AF9-transformed LSK cells from Jmjd1 $c^{f f}$ or WT controls into recipient mice to generate primary leukemias. Leukemia cells harvested from moribund animals were transduced with either Cre MSCV-IRES-Tomato (CRE-Tomato) or empty vector control virus (MIT) and sorted for GFP/Tomato double-positive cells. Loss of JMJD1C profoundly decreased expansion of leukemia cells in vitro. At day 12, there was a 50 -fold decrease in the number of Jmjd1 $c^{f / f}$ CRE-Tomato cells compared with MIT control cells. This was in contrast to a 4-fold decrease in WT control cells with or without CRE-Tomato (Supplemental Figure 2C). The colony-forming activity, used as a surrogate for assessing myeloid transformation, of Jmjd1 $c^{f / f} \mathrm{CRE}-$ Tomato cells was greatly reduced in serial replating assays (Figure $2 \mathrm{~B}$ ). The resulting colonies after 1 week were much smaller in size and more diffused in morphology. Wright-Giemsa staining of cells from these colonies confirmed that they were composed of cells that had the appearance of differentiated neutrophils/macrophages (Figure 2C). In contrast, control WT leukemia with or without CRE-Tomato did not show a lasting effect on colony number (Figure 2B) or on colony or cell morphology (Figure 2C). In addition, we observed a significant increase in apoptosis upon Jmjd1C deletion (Figure 2D). Cell-cycle analysis by BrdU labeling showed a modest but significant decrease, by $12 \%$, in the percentage of 
A

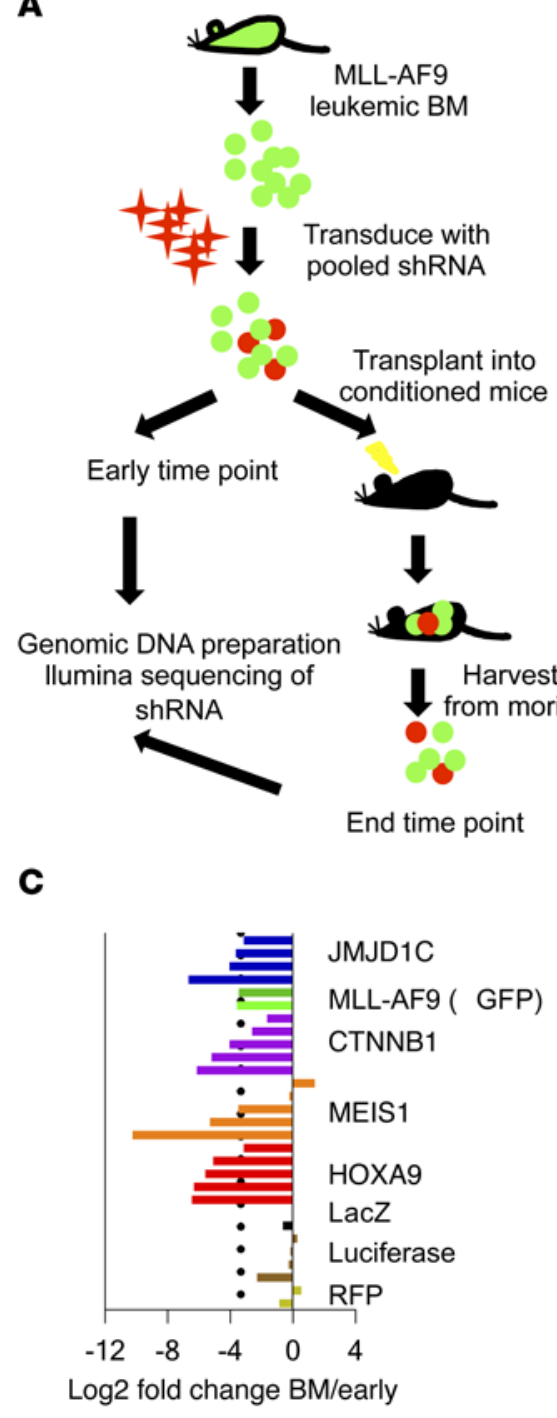

B

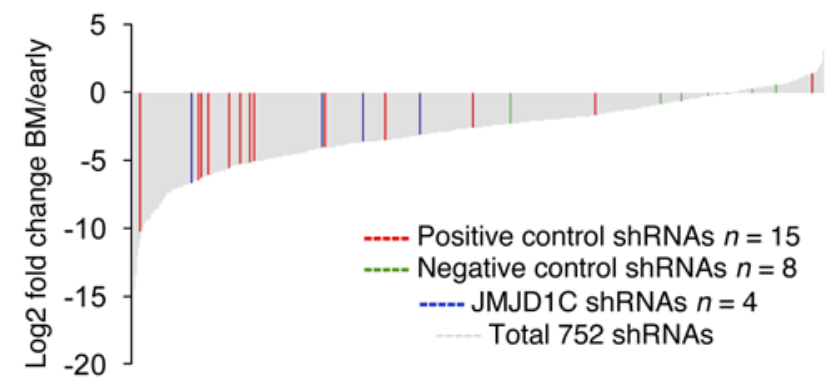

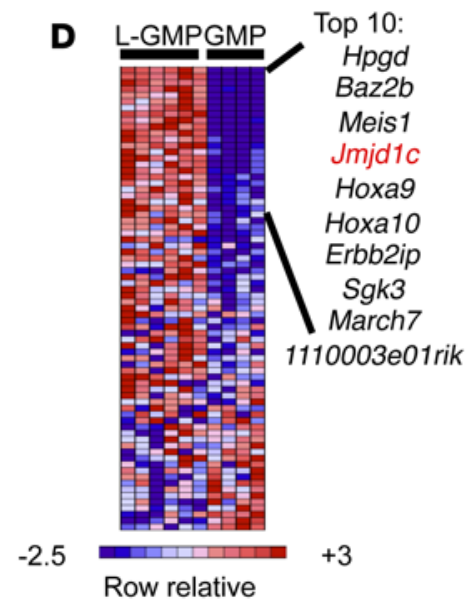

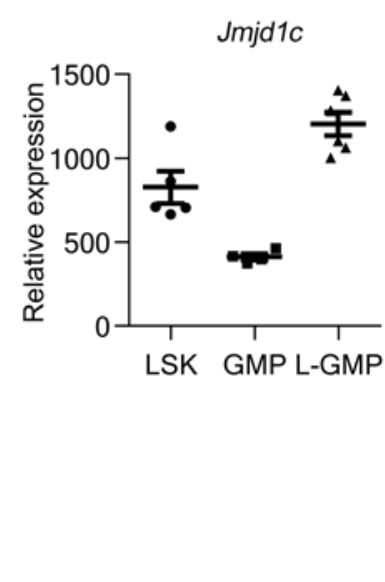

Figure 1. In vivo shRNA screening of MLL-AF9 targets identifies JMJD1C as essential for MLL-AF9 leukemia. (A) Schematics of in vivo shRNA screen. (B) Bar graph of $\log _{2}$ fold change in the BM of all hairpins used in the screen. (C) Bar graph of $\log _{2}$ fold change of hairpins against JMJD1C, showing positive (HOXA9, MEIS1, CTNNB1, MLL-AF9 [GFP]) and negative (LacZ, luciferase, and Rfp) hairpins in the screen. (D) Left panel: heat map of microarray geneexpression data (14) on MLL-AF9 target genes (28). Right panel: Jmjd1c expression data derived from microarray data (14). Data are represented as mean \pm SEM in D. See also Supplemental Figure 1 and Supplemental Tables 1 and 2.

cells in S phase (Figure 2E). Overall these data show that JMJD1C is required for continued proliferation and colony-forming activity of MLL-AF9 leukemia cells.

Loss of JMJD1C reduces LSC frequency in MLL-AF9 leukemia. Our in vitro findings prompted us to further examine the role of JMJD1C in MLL-AF9 leukemogenesis in vivo. We transduced primary LSK-derived leukemias from a Jmjd1 $c^{\text {fff }}$ background with either CRE-Tomato or MIT and sorted for GFP/Tomato doublepositive cells. We transplanted 5,000 to 300,000 cells into conditioned mice. We found that loss of JMJD1C markedly delayed AML development and prolonged survival in secondary recipient mice. Also, there was a significant decrease in leukemia-initiating cell frequency upon loss of JMJD1C (1 in 7,000 vs. 1 in 53,000) (Supplemental Figure 2D and Supplemental Table 3). The leukemias that developed from Jmjd1dff CRE-Tomato cells were completely deleted for Jmjd1c (Supplemental Figure 2D). Characterization of disease in mice that received Jmjd1 $c^{\text {ff }} \mathrm{CRE}$-Tomato cells showed that it is consistent with AML. The mice displayed leukocytosis, anemia, and thrombocytopenia with marked splenomegaly. Flow cytometry analysis of the BM and SPL from moribund animals showed replacement of BM and SPL with GFP/Tomato-positive cells with the immune phenotype of $\mathrm{Mac1}^{+} \mathrm{Gr} 1^{+} \mathrm{B}^{2} 2 \mathrm{O}^{-} \mathrm{CD}^{-}$(data not shown). The resulting leukemia from Jmjd1 $c^{f / f} \mathrm{CRE}$-Tomato recipients has a more differentiated phenotype, as evidenced by decreased c-Kit-positive cells and differentiated morphology by Wright-Giemsa staining (Supplemental Figure 2, E and F). Consistently, these $J \mathrm{mjd} \mathrm{c}^{-/-}$leukemic cells showed decreased clonogenic activity (Supplemental Figure 2G, $\sim 2.5$ - to 5.5 -fold) in vitro in CFC 
A

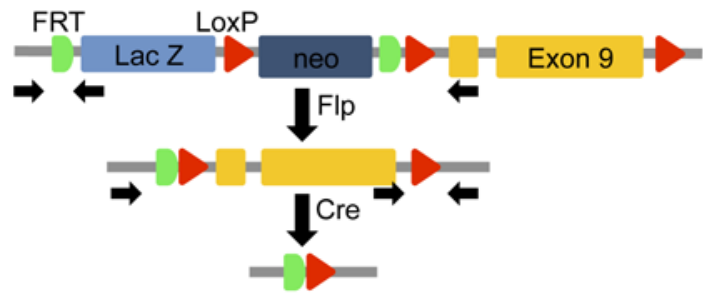

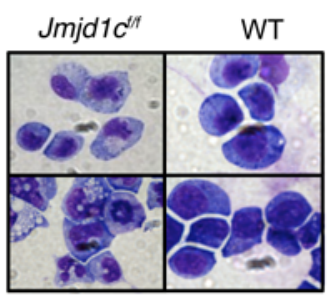

$\mathbf{F}$
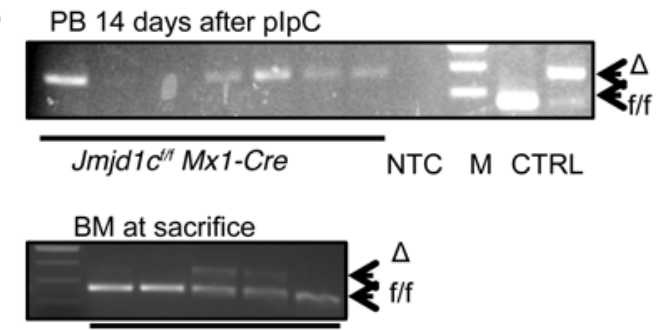

M Jmjd1c/f Mx1-Cre
B

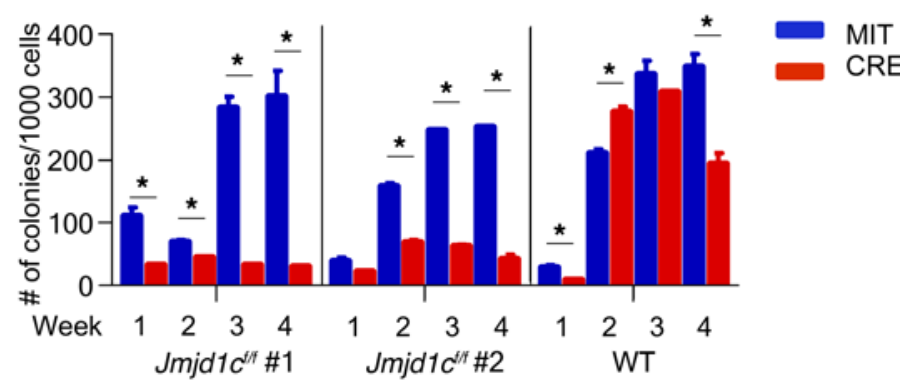

D

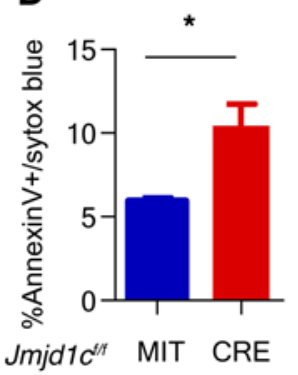

E

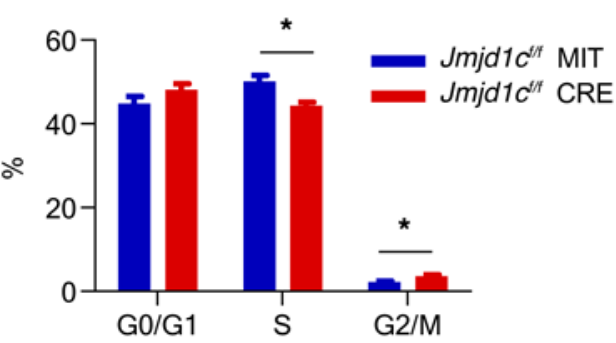

G

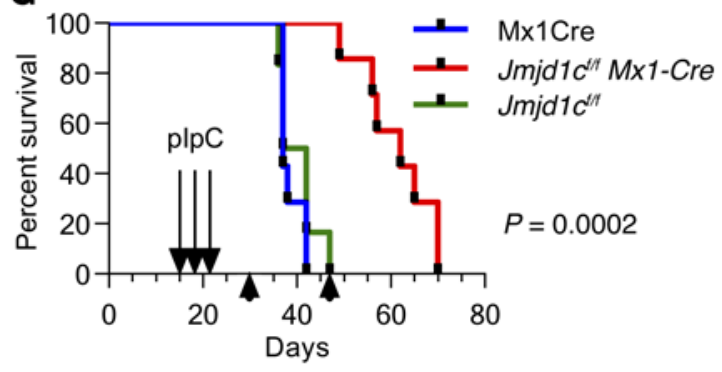

Figure 2. Loss of JMJD1C decreases LSC frequency in established MLL-AF9 leukemia. (A) Schematics of conditional knockout allele of Jmjd1c. (B) Colony counts of serial replating of primary MLL-AF9 leukemia after transduction of CRE or MIT control viruses in methylcellulose. Duplicate samples of 2 independent $/ \mathrm{mjd}_{1} \mathrm{c}^{\mathrm{f} / \mathrm{f}}$ and $1 \mathrm{WT}$ leukemia are shown. (C) Morphologic changes (left, colony in methylcellulose; right, Wright-Ciemsa stain) in established MLLAF9 leukemia 7 days after transduction with CRE. Scale bars: $100 \mu \mathrm{m}$ (left panels). Original magnification, $\times 400$ (right panels). (D and E) Flow cytometry analysis of apoptosis (D) and cell cycle by BrdU and sytox blue (E) in MLL-AF9 leukemia 6 days after transduction with CRE. Results from 3 independent leukemias for $\mathbf{D}$ and $\mathbf{E}$. (F) Genotyping result of PB day 14 after plpC and BM at the time of sacrifice. (C) Survival curve of secondary recipient mice that received $\mathrm{Mx1}$-Cre $(n=7)$, Jmjd1 $c^{f / f}(n=6)$, or $/ m j d 1 c^{f / f} \mathrm{Mx1}$-Cre $(n=7)$ MLL-AF9 leukemia after plpC administration. Data are represented as mean \pm SEM in B D, and $\mathbf{E} .{ }^{*} P<0.05$, Student's $t$ test. See also Supplemental Figure 2.

assay. Consistent with increased apoptosis in vitro, we observed significant increase in cleaved caspase 3 by Western blotting in Jmjd1 $\mathrm{c}^{-/}$leukemia compared with controls (Supplemental Figure $2 \mathrm{H})$. These data show that loss of JMJD1C leads to a significant reduction in LSC frequency and that the LSC that do remain give rise to leukemia with a more differentiated phenotype.

To assess the effect of acute loss of JMJD1C on leukemia maintenance, which better mimics potential therapeutic intervention, we generated primary leukemias by transducing LSK cells from $J m j d 1 d^{f f} \mathrm{M} x 1$-Cre, Jmjd1 $c^{f / f}$, and Mx1-Cre mice with MLL-AF9 GFP, followed by injection into primary recipient mice. Leukemia cells were harvested from moribund animals and transplanted into secondary recipient mice. When engraftment of leukemia cells was detected in peripheral blood (PB), defined by $1 \%$ to $10 \%$ PB GFP, we administered polyinosinic-polycytidylic (pIpC) to the secondary recipient mice. Deletion of Jmjd1c was confirmed by genotyping of PB 2 weeks after PIpC administration (Figure 2F). To test the effect of acute loss of JMJD1C on the clonogenic activity of the leukemic cells, we isolated $\mathrm{GFP}^{+}$cells from PB 29 days after pIpC administration and performed CFC assay. We observed a dramatic decrease in the number of colonies from $\mathrm{GFP}^{+} J m j d 1 c^{\text {f/f }}$ Mx1-Cre cells compared with control (Supplemental Figure 2I). Loss of Jmjd1c in this setting significantly prolonged survival of Jmjd1c/f Mx1-Cre mice compared with controls (Figure 2G). When the mice from the pIpC-treated Jmjd1 d/f Mx1-Cre group eventually succumbed to the disease, genotyping results showed that the leukemia cells were composed of cells that had escaped complete deletion of Jmjd1c (Figure 2F), suggesting a strong selection pressure against losing JMJD1C. Together, these results demonstrated that JMJD1C prevents differentiation and apoptosis of leukemia cells and thus is important for maintaining LSCs in MLL-AF9 leukemia. To study the effect of loss of JMJD1C on human leukemia cells, we used an shRNA approach in human leukemia cell lines and our results showed that loss of JMJD1C had a more profound 
A
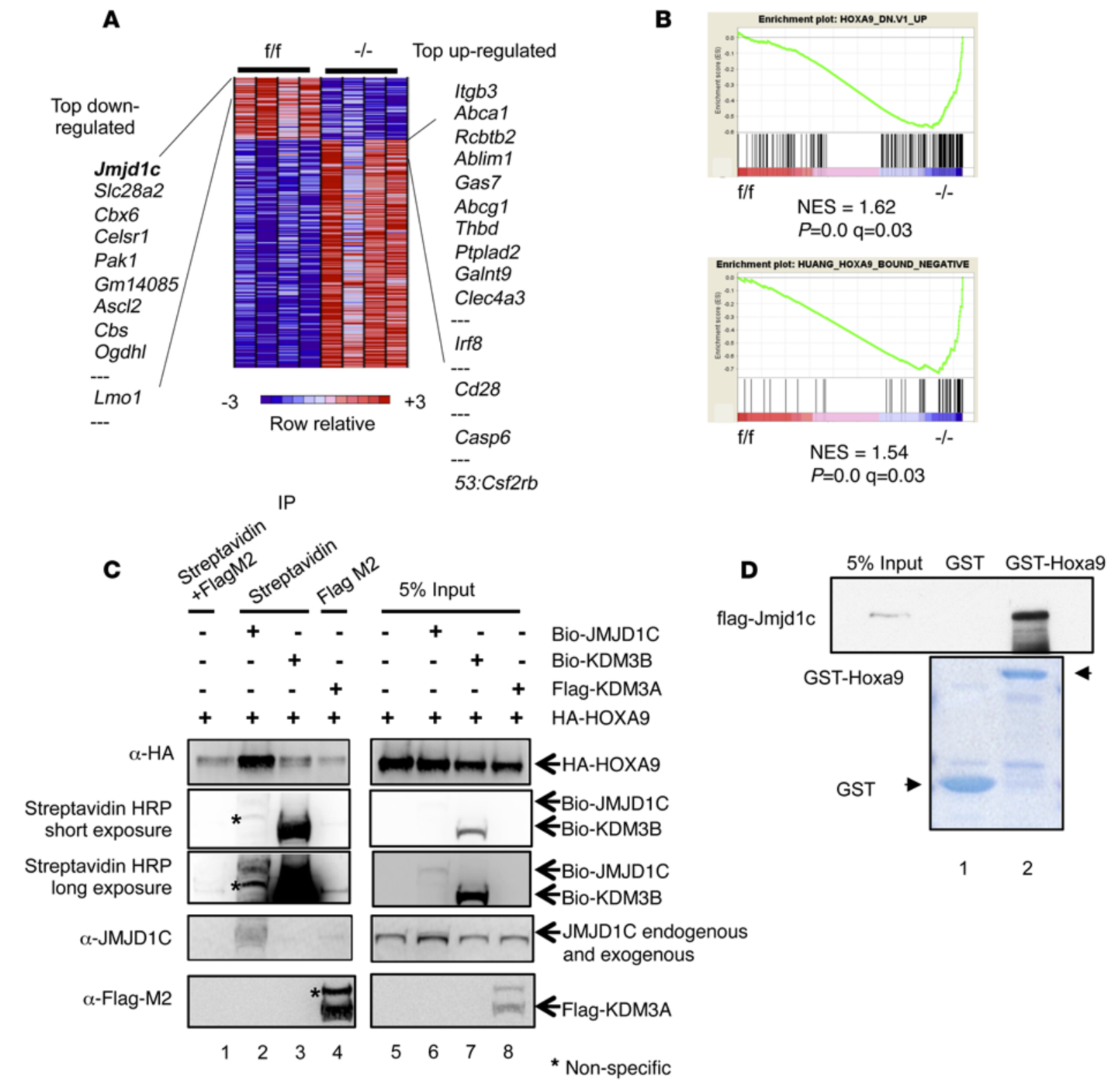

.

Figure 3. JMJD1C interacts with HOXA9 and modulates a HOXA9-controlled gene-expression program. (A) Heat map of differentially expressed genes in MLL-AF9 leukemia cells 6 days after loss of JMJD1C. (B) CSEA analysis result showing enrichment of HOXA9 repressed genes (37) and HOXA9 bound and repressed genes (44). NES, normalized enrichment score. (C) Co-IP of HA-HOXA9 and KDM3A family members in 293T cells transfected with indicated plasmids. (D) GST-binding assay using bacteria expressed and purified GST-HOXA9 and baculovirus system expressed and purified flag-JMJD1C. See also Supplemental Figure 3 and Supplemental Table 4.

effect on MLL-rearranged than non-MLL-rearranged cells (Supplemental Figure 2, L-N).

To investigate whether JMJD1C is also important for MLLAF9 leukemia initiation, we transduced LSK cells from Jmjd1c/f Vav1-Cre mice, which have complete deletion of Jmjd1c in this compartment (see below), and compared the colony-forming activity of Jmjdc1-deficient LSK cells with that of control LSK cells in the CFC assay. The result showed that loss of JMJD1C only transiently affected the colony-forming ability of the cell; an approximately 3.5-fold reduction was observed at weeks 2 and 3 , with no reduction at week 4 (Supplemental Figure 2J). Consistent with this result, when we transplanted the same cells into conditioned mice, recipient mice succumbed to leukemia with a similar median survival compared with controls (Supplemental Figure 2K). Together, these data suggest that JMJD1C is important for leukemia maintenance in both human and mouse MLLAF9 leukemia, but is dispensable for leukemia initiation.

JMJD1C modulates HOXA9-controlled gene expression. To understand the mechanism underlying JMJD1C function in MLL-AF9 leukemogenesis, we examined gene-expression changes in MLL-AF9 leukemias after loss of JMJD1C. Leukemic BM from mice transplanted with Jmjd1 $c^{f / f}$ MLL-AF9 GFP was transduced with retroviral-CRE-Tomato or control retroviral-Tomato viruses. GFP/Tomato double-positive cells were sorted at day 6 after transduction. RNA was purified, amplified, and subjected to RNA sequencing (RNA-seq). We identified 57 

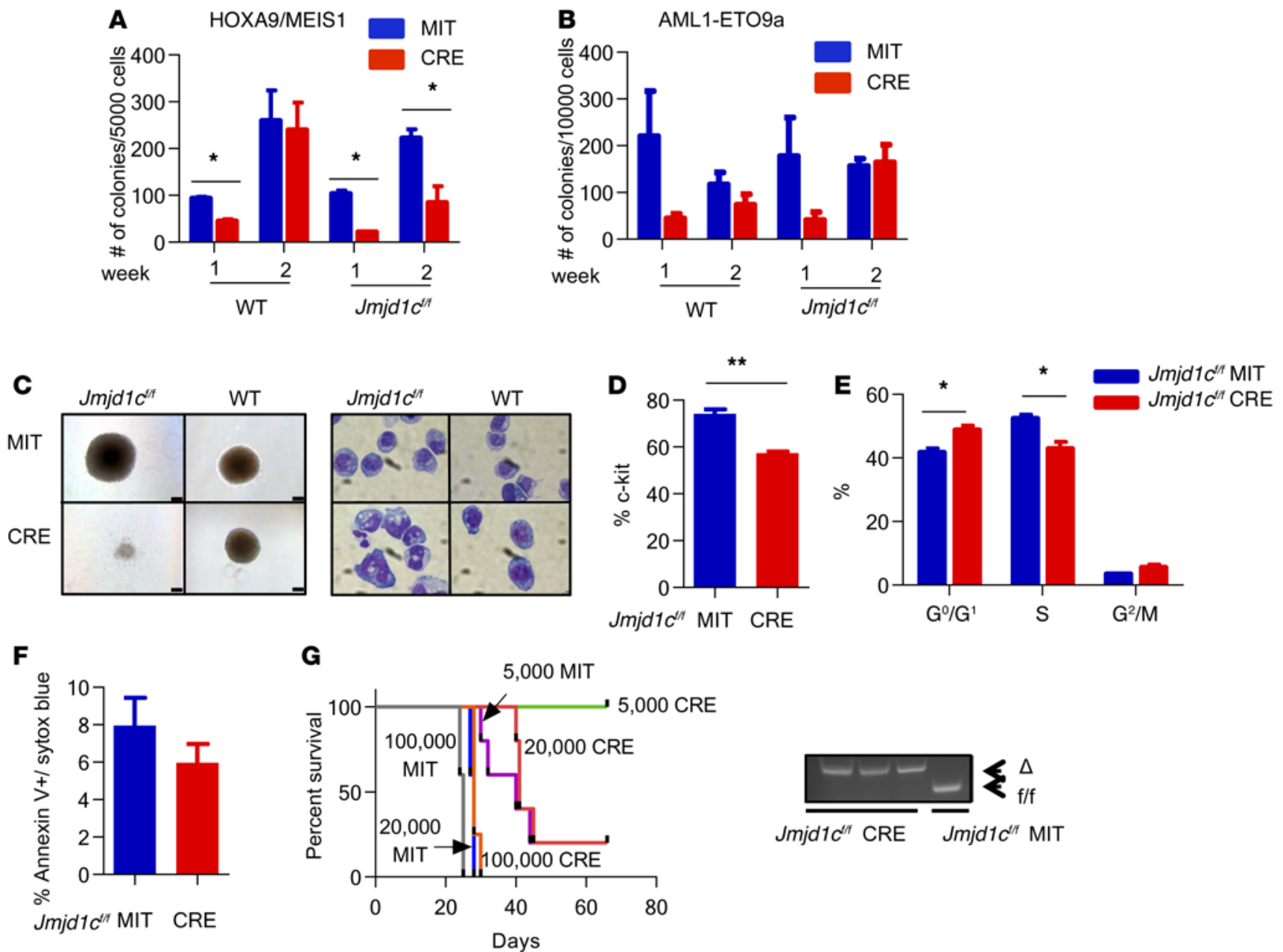

Figure 4. Loss of JMJD1C impairs leukemic transformation by HOXA9/MEIS1. (A) Colony counts of HOXA9/MEIS1 transformed LSKs from /mjd1c/f BM after transduction of CRE or MIT control viruses in methylcellulose. (B) Colony counts of AML-ETO9a transformed LIN ${ }^{-} / m j d 1 c^{f / f}$ BM cells after transduction of CRE or MIT control viruses in methylcellulose. Results from 2 to 3 independent experiments for A and B. (C) Morphologic changes (left, colony in methylcellulose; right, Wright-Ciemsa stain) in HOXA9/MEIS1 preleukemia cells 10 days after transduction with CRE. Scale bars: $100 \mu$ m (left panels). Original magnification, $\times 400$ (right panels). (D-F) Flow cytometry analysis of c-Kit expression (D), cell-cycle analysis by BrdU and sytox blue (E), apoptosis analysis (F) in HOXA9/ MEIS1 leukemic cells day 5 after transduction with CRE. Results from 3 independent leukemias. (C) Survival curves of secondary recipient mice that received 100,$000 ; 20,000$ or 5,000 double-sorted GFP+Tomato ${ }^{+}$HOXA9/MEIS1 leukemia cells 2 days after transduction with CRE or MIT control viruses ( $n=5$ per group). Right panel: genotyping result of BM at the time of sacrifice. Data are represented as mean \pm SEM in $\mathbf{A}$, B, and $\mathbf{D}-\mathbf{F}$. ${ }^{*} P<0.05 ;{ }^{* *} P<0.01$ Student's $t$ test.

downregulated and 211 upregulated genes using a criteria of a greater than 1.5 -fold change and $P<0.05$ (Figure 3A and Supplemental Table 4). Gene Set Enrichment Analysis (GSEA) $(42,43)$ showed that myeloid differentiation and apoptosis signatures were enriched in $J m j d 1 c^{-/-}$samples (Supplemental Figure 3A). This is consistent with the observed effect of increased apoptosis and induced differentiation upon JMJD1C loss in MLL-AF9 leukemia. Strikingly, when we subjected the RNA-seq data to GSEA analysis using a collection of oncogenic signatures from the Molecular Signatures Database (MSigDB) (42), the top ranking signature in the $\mathrm{Jmj} \mathrm{d1c^{-1 }}$ samples was from a set of genes that are upregulated upon HOXA9 suppression in Molm13 cells (37). This finding was corroborated by the enrichment of a set of genes that were bound and negatively regulated by HOXA9 in murine BM transduced by HOXA9/MEIS1 (refs. 44, 45, and Figure 3B). Therefore, JMJD1C is required for a gene-expression program that is also maintained by HOXA9.
Based on this finding, we hypothesized that JMJD1C may be a cofactor for HOXA9 and thus modulate HOXA9-mediated gene expression. To test this hypothesis, we examined whether JMJD1C and HOXA9 physically interact. We transiently coexpressed KDM3 family members (biotin-tagged JMJD1C and KDM3B where BirA was expressed in the bicistronic IRES-BirA transcript and Flag-tagged KDM3A) and HA-tagged HOXA9 in 293T cells. We observed low-level expression of JMJD1C, which may have been due to the large size of the protein, 2,540 amino acids (Figure 3C). Nonetheless, we detected specific interaction between HOXA9 and JMJD1C, but not other members of the KDM3 family, in the coimmunoprecipitation (co-IP) experiment (Figure 3C). Moreover, we transiently expressed JMJD1C in 293T and examined the interaction between JMJD1C and endogenous HOXA10, another homeobox transcription factor that is also implicated in leukemia development (46), but did not detect any specific interaction (Supplemental Figure 3B). These data suggest that the 2 
Table 1. Limiting dilution assay of HOXA9/MEIS1 leukemia upon loss of JMJD1C

\begin{tabular}{lccc} 
Dose & \multicolumn{2}{c}{ Response/tested } & $P$ value \\
& MIT & CRE & \\
100,000 & $5 / 5$ & $4 / 4$ & 0.0067 \\
20,000 & $5 / 5$ & $4 / 5$ & 0.0031 \\
5,000 & $4 / 5$ & $0 / 5$ & 0.0133 \\
LIC frequency & 1 in 3,062 & 1 in 19,006 &
\end{tabular}

LIC, leukemia-intiating cell.

proteins interact directly with each other or exist in a complex. To test whether there is direct protein-protein interaction between JMJD1C and HOXA9, we expressed and purified Flag-tagged JMJD1C using a Baculovirus expression system and used it in GST pull-down assays with GST-HOXA9 or GST alone. We observed a specific interaction between GST-HOXA9 and Flag-JMJD1C (Figure 3D). Similarly, we showed specific interaction between purified HA-HOXA9 and Flag-JMJD1C (Supplemental Figure 3B).

Loss of JMJD1C reduces LSC frequency in HOXA9/MEIS1 leukemia. Given our observation that JMJD1C plays a role in HOXA9associated gene expression, we reasoned that loss of JMJD1C would also impair leukemogenesis of HOXA9/MEIS1-driven leukemia (47). To test this, we performed CFC assays on HOXA9/ MEIS1-transformed LSK BM cells. We found that loss of JMJD1C reduced the number of colonies in Jmjd1 $c^{f / f} \mathrm{CRE}$-Tomato versus MIT compared with WT control, much as we had seen for MLLAF9 (Figure $4 \mathrm{~A})$ in serial replating $(P<0.05$ comparing WT CRE-Tomato sample to that of $\left.J m j d 1 c^{f / f}\right)$. The colonies in the CRETomato samples were smaller in size and were composed of differentiated cells that resembled neutrophils and macrophages by Wright-Giemsa staining (Figure 4C). This is in contrast to another histone-modifying enzyme, DOT1L, which is essential for MLLAF9 leukemia, but is dispensable for HOXA9/MEIS1-driven leukemia because Dot1l controls endogenous HOXA9/MEIS1 expression and has no effect on exogenous retroviral-driven HOXA9/MEIS1 leukemia (28). As a control, we performed CFC assays with BM cells transformed with AML1-ETO9a (48), which does not express HOXA9. We did not observe decreases in colony numbers in Jmjd1 $d^{f / f} \mathrm{CRE}$-Tomato versus MIT compared with WT controls (Figure 4B). To interrogate the effect of losing JMJD1C on HOXA9/MEIS1 leukemia, we transduced LSK-derived primary leukemias generated in the Jmjd1 $c^{f / f}$ background with either CRE-Tomato or MIT control to delete Jmjd1c. Deleting Jmjd1c in the leukemia cells resulted in decreased c-Kit expression (Figure 4D) and decreased proliferation of leukemic cells, as evidenced by a decreased proportion of cells in the $\mathrm{S}$ phase concomitant with an increase in the $\mathrm{G}_{1}$ phase (Figure $4 \mathrm{E}$ ). In contrast to MLLAf9 leukemia, loss of JMJD1C in HOXA9/MEIS1 leukemia did not increase apoptosis of the cells (Figure $4 \mathrm{~F}$ ). To further investigate the effect of losing JMJD1C in vivo, we sorted GFP/Tomato double-positive cells after transduction of CRE-Tomato, followed by transplantation into conditioned mice. Deletion of Jmjd1c was confirmed by genotyping of the leukemic BM cells at end point (Figure 4G). Loss of JMJD1C significantly delayed disease pro- gression and prolonged survival in secondary recipient mice at 3 dilutions, which is consistent with an effect on LSC (Figure 4G). There was also a significant decrease in leukemia-initiating cell frequency upon loss of JMJD1C (1 in 3,062 vs. 1 in 19,006, Table 1). We next examined whether JMJD1C is important for leukemia initiation in this model. Similar to what we have observed in MLLAF9 leukemia, loss of JMJD1C resulted in only transient reduction in colony numbers in serial replating assay (Supplemental Figure 2O, 1.5-fold at week 2), suggesting that JMJD1C is not required for HOXA9/MEIS1 leukemia initiation.

Next, we carried out gene-expression profiling by RNA-seq in HOXA9/MEIS1 leukemias upon loss of Jmjd1c similar to that in MLL-AF9 leukemias. A total of 38 genes were downregulated and 380 upregulated using criteria of greater than 1.5 -fold change and $P<0.05$ (Figure 5A and Supplemental Table 5). Consistent with the observed effect of increased differentiation and decreased cellcycle progression, GSEA analysis showed that myeloid differentiation and negative regulation of cell-cycle signatures were enriched in Jmjd1 $c^{-/-}$samples (Supplemental Figure 3E). Strikingly, the top ranking signature in the Jmjd1 $c^{-/}$HOXA9/MEIS1 samples was the same as that in Jmjd1c $c^{-1-}$ MLL-Af9: a set of genes that are upregulated upon HOXA9 suppression in Molm13 cells (ref. 37 and Figure 5B). Similar to that in MLL-AF9, we found enrichment of a set of genes bound and negatively regulated by HOXA9 in murine BM transduced by HOXA9/MEIS1 (refs. 44, 45, and Figure 5B). Moreover, we found a significant number of overlap between genes that were upregulated upon loss of JMJD1C in MLL-AF9 (74/211) and HOXA9/MEIS (74/380) leukemic cells (Figure 5C). These results suggested that JMJD1C is required for maintaining a gene-expression program in both MLL-AF9 and HOXA9/MEIS1 leukemia. Together, our results show that JMJD1C is important for leukemia that depends on HOXA9 and further corroborate our hypothesis that JMJD1C is important for HOXA9-mediated gene expression.

JMJD1C is dispensable for steady-state hematopoiesis. The expression of JMJD1C is highest in immature hematopoietic stem and progenitor cells (HSPC) and decreases during differentiation (Supplemental Figure 4A). To assess the role of JMJD1C in normal hematopoiesis in vivo, we crossed Jmjd1 d/f with Vav1-Cre mice (49) to delete Jmjd1c in hematopoietic compartments. Jmjd1c/f Vav1-Cre mice were born with normal Mendelian frequency (data not shown). Complete deletion of Jmjd1c was observed in LSKs with near complete deletion of Jmjd1c in the BM and SPL in 6-week-old mice (Figure 6A). Analysis of the complete blood counts (CBCs) from 6- to 8-week-old mice showed no difference between Jmjd1c/- and Vav1Cre control mice (Figure $6 \mathrm{~B})$. A significant reduction in BM $(20 \%$, $P=0.02)$ but not SPL cellularity $(\sim 40 \%, P=0.1)$ was observed (Figure $6 \mathrm{C}$ ) from age- and sex-matched 6- to 8-week-old mice. When the HSPC compartment was analyzed, we observed a modest decrease in the total number of granulocyte-macrophage progenitor (GMP), common myeloid progenitor (CMP), and common lymphoid progenitor (CLP) (ranging from 30\%-50\%), but not of HSC (defined by CD150 ${ }^{+} \mathrm{CD} 48^{-} \mathrm{LSK}$ ) in the BM (Figure 6D, schematics of the gates are shown in Supplemental Figure 4B). To investigate the effect of loss of JMJD1C on progenitor function, we isolated BM cells from Vav1-Cre and Jmjd1 $c^{f / f}$ Vav1-Cre mice and subjected them to CFC assays under conditions for myeloid colony formation. We did not observe any significant change in the CFC numbers or phe- 
A

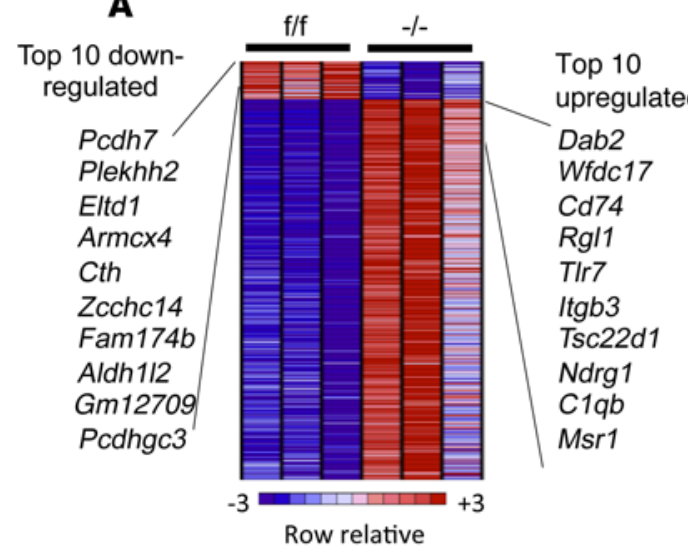

C

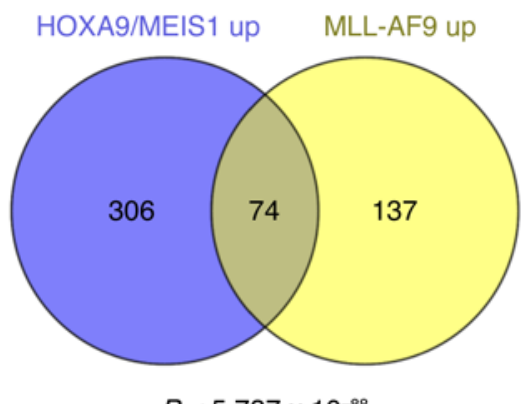

$P<5.737 \times 10^{-88}$
B

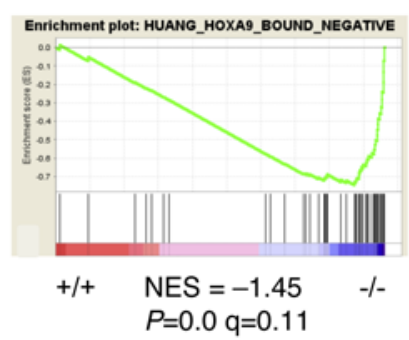

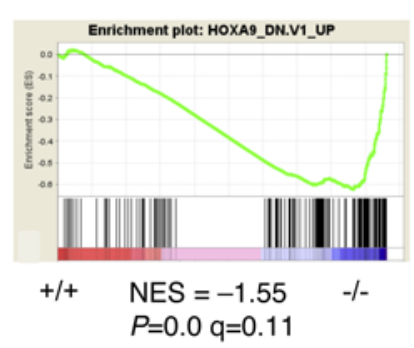

Figure 5. Loss of JMJD1C affected a HOXA9-controlled gene-expression program in HOXA9/MEIS1 leukemia. (A) Heat map showing differentially expressed genes in HOXA9/MEIS1 leukemia cells 6 days after deleting Jmjd1c. (B) GSEA analysis result showing enrichment of HOXA9 repressed genes (37) and HOXA9 bound and repressed genes (44). See also Supplemental Table 5. (C) Venn diagram of overlapping genes that are upregulated upon loss of JMJD1C in MLLL-AF9 and HOXA9/ MEIS1 leukemia cells. $P$ value calculated by exact hypergeometric probability.

at 18 weeks (Figure 7B). Analysis of the HSPC compartments showed a significant decrease of chimerism in the LSK, GMP, and CMP compartments (Figure 7C). Serial transplantation of primary BM into secondary recipients showed a similar result (Figure 7, D-F) with a trend toward decreased chimerism in HSPC compartments. This result was confirmed in primary competitive transplantation experiments using HSCs $\left(\mathrm{CD}_{150}{ }^{+} \mathrm{CD} 48^{-} \mathrm{LSK}\right)$ as donor cells (Supplemental Figure 5, J-L) where we also observed a significant, but mod-

notypes (Supplemental Figure 4C). Furthermore, the total number of Mac-1/Gr-1 cells in the BM and SPL was not altered (Figure 6E). There was a slight decrease $(-30 \%-40 \%)$ in the number of $\mathrm{B} 220^{+}$ cells in the BM and SPL and an approximately $40 \%$ reduction of $\mathrm{CD}^{+}$cells in the SPL (Figure 6E). These results demonstrate that JMJD1C is largely dispensable for steady-state hematopoiesis.

Loss of JMJD1C impairs cell-autonomous HSPC regenerative function. We next assessed the function of JMJD1C in hematopoietic regeneration by transplanting CD45.2 marked Jmjd1 f/f Vav1-Cre (referred to as $\mathrm{Jmj} \mathrm{dc}^{-/}$) or Vav1-Cre BM cells into lethally irradiated CD45.1 B6.SJL recipient mice in a noncompetitive setting. Mice transplanted with $J m j d 1 c^{-/-}$whole BM showed efficient longterm reconstitution of the hematopoietic system similar to that of the control. This is evidenced by a high percentage of CD45.2 cells in Jmjd1c $c^{-/}$recipient mice in PB over the course of 18 weeks (Supplemental Figure 5A). Also, reconstitution of BM, SPL, and HSPC compartments with CD45.2-positive Jmjd1c $c^{-/}$cells was greater than $90 \%$ at 18 weeks (Supplemental Figure 5, B and C). Serial transplantation of primary BM into secondary recipients showed similar results (Supplemental Figure 5, D-F) with a slight decrease in $\mathrm{PB}$ chimerism, indicating intact self-renewal ability of HSC. We then tested the effect of loss of JMJD1C on the competitiveness of HSPCs by transplanting CD45.2-marked Jmjd1 1/or Vav1-Cre BM cells with CD45.1 BM cells from B6.SJL mice at a 1:1 ratio. Mice transplanted with $J m j d 1 c^{-/-}$cells showed a marked decrease in PB chimerism in all lineages as early as 4 weeks that persisted throughout 18 weeks compared with those that received Vav1-Cre BM (Figure 7A and Supplemental Figure 5, G-I). We also observed a significant decrease in BM and SPL chimerism est, decrease in HSC chimerism, by $25 \%$, as well as decreased chimerism in progenitor compartments. Together, these results show that although $\mathrm{Jmjd}_{1} \mathrm{c}^{-/-} \mathrm{HSCs}$ can fully reconstitute hematopoiesis systems following transplantation, they do have decreased competitiveness compared with controls.

To address the question of whether the decreased engraftment we observed in transplantation is due to a deficiency in homing of $J m j d 1 c^{-/-}$cells, we tested homing of $J m j d 1 c^{-/-}$cells by analyzing BM chimerism 18 hours after transplantation. We found BM CD45.2 levels were similar between mice that received Jmjd1 $\mathrm{c}^{-/-}$ cells and those that received control cells (Supplemental Figure $5 \mathrm{M})$, suggesting no defect in the homing ability of $J m j d 1 \mathrm{c}^{-}$cells. In addition, we performed noncompetitive and competitive repopulating experiments using Jmjd1 $c^{f / f} \mathrm{Mx1}$-Cre mice. We transplanted Jmjd1 $c^{f / f}$ Mx1-Cre or Mx1-Cre BM cells alone or mixed 1:1 with CD45.1 BM cells into lethally irradiated CD45.1 B6.SJL recipient mice, waited for stable engraftment (4 weeks), and then induced deletion of Jmjd1c by pIpC administration. Since the cells were uninduced prior to transplantation, they displayed similar levels of engraftment at week 4 after transplantation, as expected (Supplemental Figure 6, A and D). We obtained similar results to what we found for Jmjd1 $c^{f / f} \mathrm{Vav1}$-Cre transplantation experiments (Supplemental Figure 6, A-F) in both noncompetitive and competitive transplantations. These results show that, independently of the Cre strain and the time of excision, Jmjd1 $\mathrm{c}^{-/-}$cells can support hematopoiesis, but that there is a defect in competitive transplantation that is not due to impaired homing ability.

To begin to understand the mechanisms underlying JMJD1C's effects in normal hematopoiesis, we performed gene-expression 
A

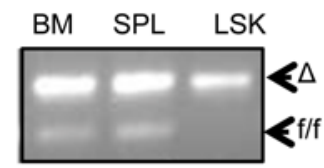

B : Vav1-Cre

- Jmjd1 $C^{\prime \prime}$ Vav1-Cre

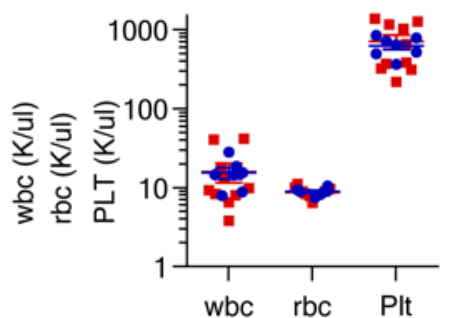

C : Vav1-Cre

- Jmjd1 1" Vav1-Cre

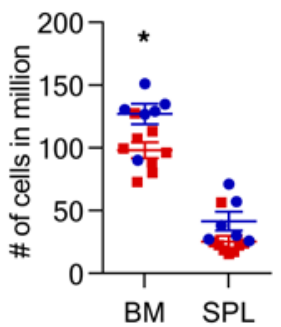

D

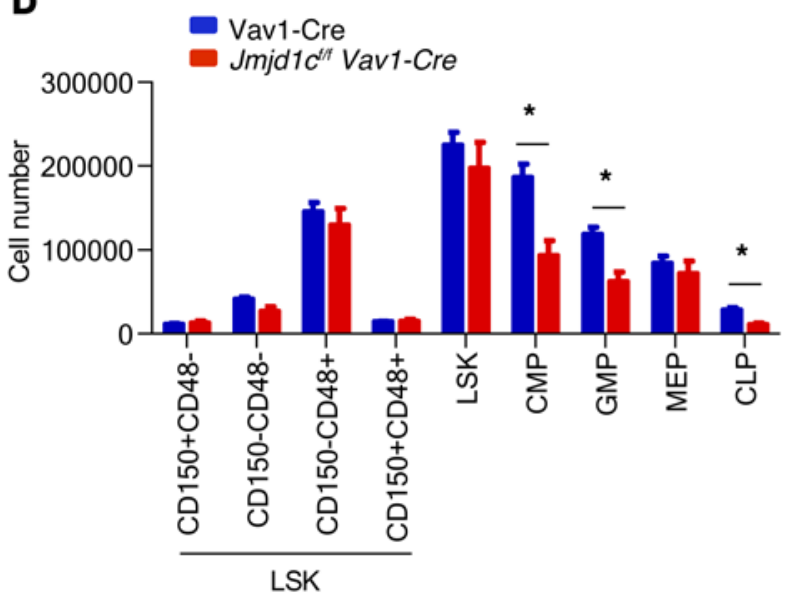

E

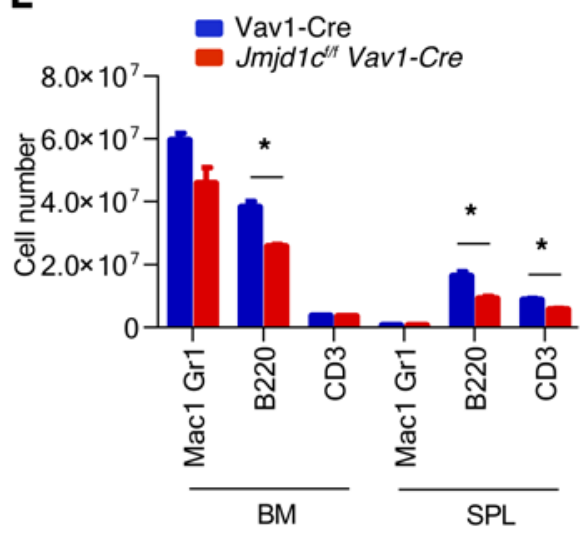

Figure 6. Effect of loss of JMJD1C in steady-state hematopoiesis. (A) Genotyping result of BM, SPL, and LSK (Lin-Sca-1+ c-Kit') cells from /mjd1c/f Vav1-Cre mice. (B) PB counts and (C) BM (from tibias, femurs, and pelvic bones) and SPL cellularity of 6- to 8-week-old Vav1-Cre and Jmjd1c/f Vav1-Cre mice. (D and E) HSPC (D) and mature cell (E) numbers in 8-week-old Vav1-Cre and Jmjd1 f/f Vav1-Cre mice $(n=3-4$ per genotype ). Data are represented as mean \pm SEM in B-E. ${ }^{*} P<0.05$, Student's $t$ test. See also Supplemental Figure 4.

RNA-seq experiments on LSK cells purified from Jmjd1cfff Vav1-Cre and Vav1-Cre. We identified 15 downregulated and 54 upregulated genes using criteria of greater than 1.5 -fold change and $P<0.05$ (Figure 7G and Supplemental Table 6). GSEA analysis showed positive enrichment of proliferation signatures, including Myc, E2F1, and cyclin D1 signatures, in Jmjd1c $c^{-/-}$LSK (Figure 7H). In agreement with this finding, we observed a significant increase in proportion of cycling cells in the HSC $(P=0.04)$ compartment with a trend toward an increase in the LSK compartment $(P=0.07)$ in JMJD1C-deficient mice compared with controls (Figure 7I). Consistently, in vivo BrdU labeling showed an increase in LSK proliferation. This was evidenced by an increase by $40 \%$ in the $\mathrm{S}$ phase $(P=0.05)$ (Supplemental Figure $5 \mathrm{~N})$. In addition, we observed a significant increase in apoptosis ( 2 -fold) in the LSK compartment, with a trend toward an increase in the HSC compartment (Figure 7K). Increased proliferation has been suggested as resulting in stem cell exhaustion and failure of long-term reconstitution of the blood systems $(50,51)$. To further address this question, we evaluated hematopoietic recovery after injection of 5-fluorouracil (5-Fu) as an alternative way to stress HSC. The results showed that $J m j d 1 c^{-/-}$mice lacked a hyperregenerative response after 5 -Fu injection, as demonstrated by diminished rebound in the level of leukocytes compared with that in control mice (Figure 7J, 50\% lower at week 2). This deficiency is not persistent, since the leukocyte level returned to normal by week 3 . Repeated 5-Fu injection showed similar results (Figure 7J, week 8-12). Based on these results, we reasoned that $J m j d 1 c^{-/-}$HSCs would exhaust faster in response to serial 5-Fu challenge. To our surprise, we did not observe any difference in survival curve between $\mathrm{Jmj}_{\mathrm{m}} 1 \mathrm{c}^{-/}$and control mice after weekly 5-Fu treatment (Supplemental Figure 5P). Moreover, when we examined the frequency and number of HSPCs 14 days after a single 5-Fu treatment, at which point HSPC numbers increased significantly (52), we observed a trend in increased frequency $(P=0.06)$ and number of LSK cells $(P=0.1)$ (Figure $7 \mathrm{~L}$ and Supplemental Figure 5O). Given that we had observed decreased $\mathrm{CBC}$ counts at the same time point after $5-\mathrm{Fu}$ injection (Figure 7J), these results would be consistent with impaired HSPC differentiation rather than exhaustion. This is also in agreement with our observation, that in competitive transplant, chimerism of JMJD1Cnull HSPC is invariably higher than that of the mature cells (Figure 7, A-F; Supplemental Figure 5, J-L; and Supplemental Figure 6, D-F). Taken together, these data suggest that loss of JMJD1C increased proliferation and apoptosis of HSPC cells and impaired its differentiation ability, resulting in diminished hematopoietic reconstitution in response to stress and decreased competitiveness.

\section{Discussion}

Epigenetic regulators play an important role in the development of cancer, and there is marked excitement that dysregulated epigenetic mechanisms represent a new therapeutic opportunity for cancer treatment (53-56). In this study, we demonstrate that JMJD1C 

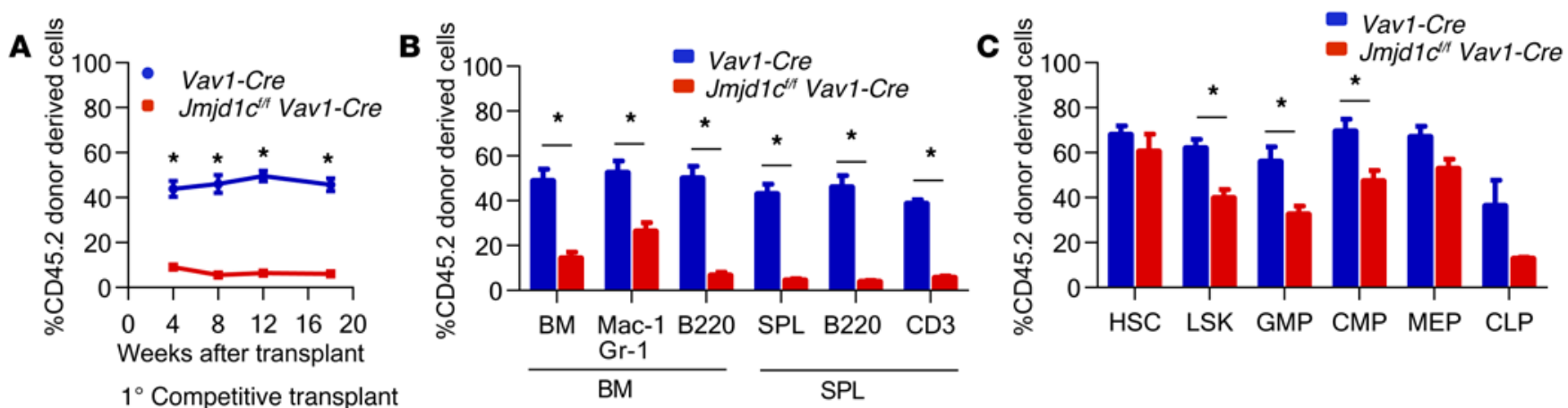

$1^{\circ}$ Competitive transplant
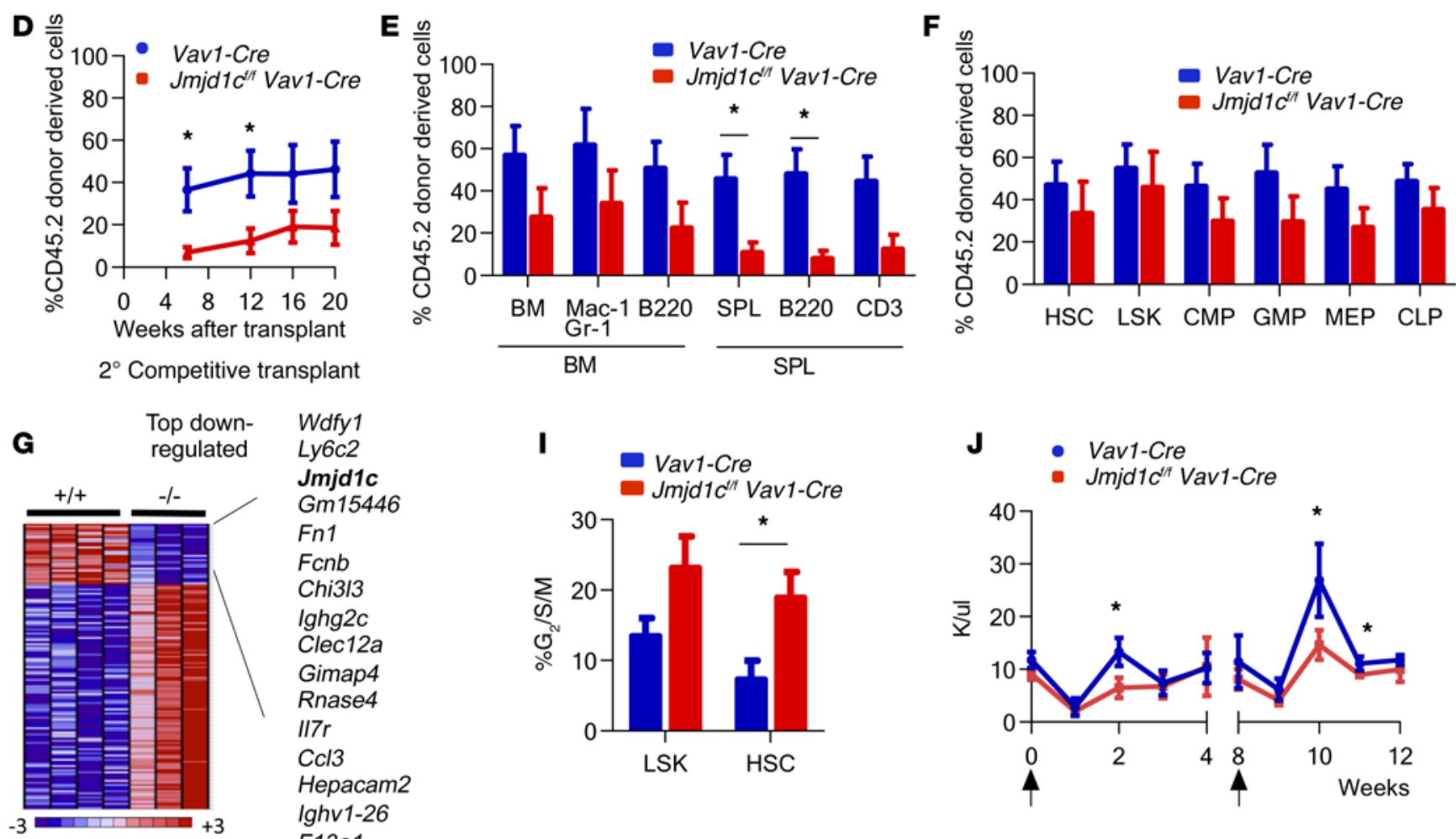

Wdfy1
Ly6c2
Jmjd1c
Gm15446
Fn1
Fcnb
Chi3/3
Ighg2c
Clec12a
Gimap4
Rnase4
II7r
Ccl3
Hepacam2
Ighv1-26
F13a1
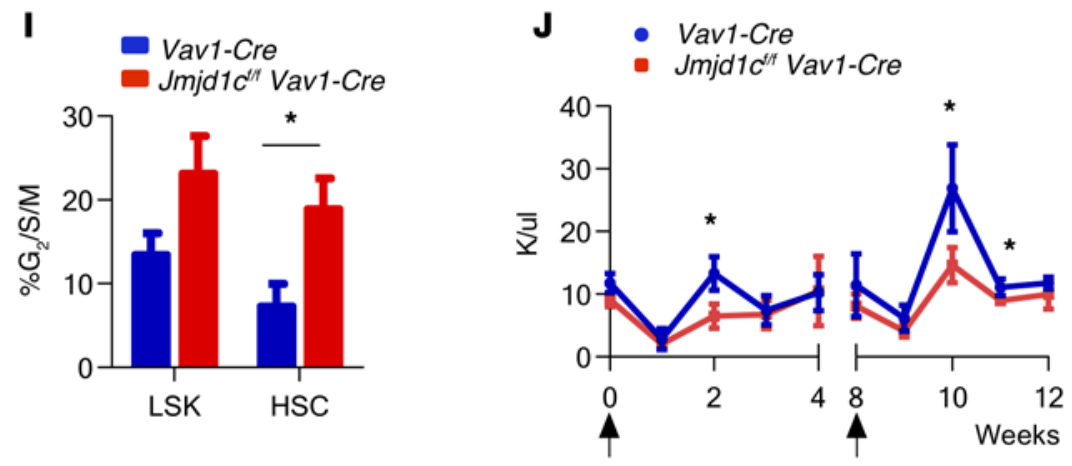

Row relative
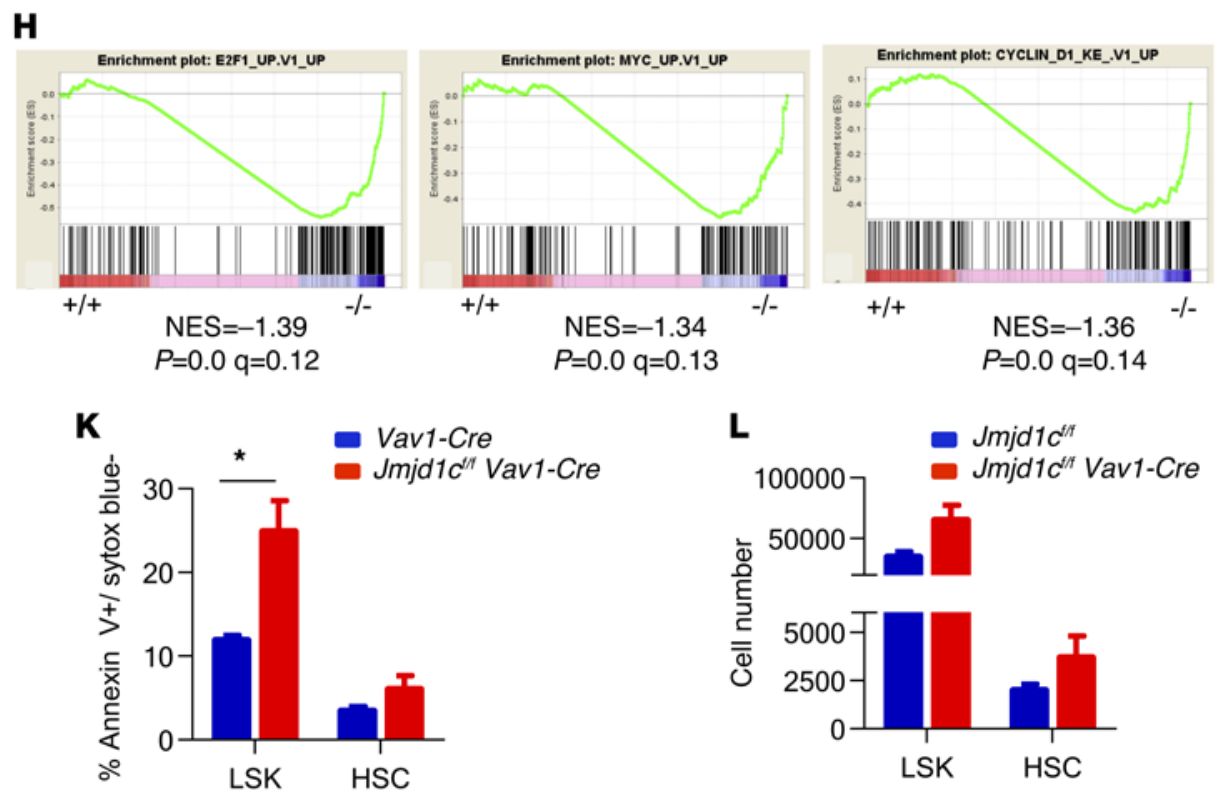
Figure 7. Effect of loss of JMJD1C on regenerative function of HSC. (A-F) Flow cytometry analysis shows results of chimerism of recipient mice from serial transplantation experiments. (A-C) One million BM cells from Vav1-Cre or Jmjd1 ${ }^{f / f}$ Vav1-Cre mice were mixed 1:1 with CD45.1 BM and transplanted into lethally irradiated CD45.1 recipients. $(n=10)$. (D-F) Two million BM cells from primary competitive transplantation were serially transplanted into secondary CD45.1 recipients $(n=5)$. Chimerism in PB (A) and (D); BM and SPL (B) and (E); stem and progenitor populations in BM (C) and (F). (C) Heat map of differentially expressed genes in Vav1-Cre and /mjd1 ${ }^{f / f}$ Vav1-Cre LSK cells. (H) GSEA analysis result showing enrichment of E2F1, Myc, and cyclin D1 signature. (I) Percentage of cells in $S / G_{2} / M$ phase of cell cycle determined by Sytox blue staining ( $n=3$ per genotype). (J) Time course of wbc counts after 5-Fu administrations (indicated by arrows, $n=5$ per genotype). (K) Percentage of apoptotic cells determined by annexin $V$ staining ( $n=3$ per genotype). (L) HSPC numbers 2 weeks after 5-Fu treatment ( $n=3-4$ per genotype). Data are represented as mean \pm SEM in A-F and I-L. ${ }^{*} P<0.05$. See also Supplemental Figures 5 and 6 and Supplemental Table 6.

differentially affects self-renewal of LSCs and HSCs. Loss of JMJD1C significantly reduced the LSC frequency in MLL-AF9 and HOXA9/MEIS1 leukemias, while its loss had a minimal effect on steady-state hematopoiesis. These findings identify JMJD1C as a potential therapeutic target in AML.

Our finding that JMJD1C is required for MLL-AF9 leukemia maintenance is consistent with the result from Sroczynska et al. (36). However, due to the different systems used, hairpin, which usually generates hypomorphic phenotypes, versus genetic knockout, which is complete null, we observed more evidence for differentiation of the leukemia cells upon loss of JMJD1C. Increased differentiation was evidenced by changes in cell and colony morphology (Figure 2C, Supplemental Figure 2, B and F), $\mathrm{c}$-Kit expression (Supplemental Figure 2E), and enrichment of differentiation signature (Supplemental Figure 3A) upon loss of JMJD1C. While Sroczynska et al. (36). did show that knockdown of JMJD1C resulted in increased expression of differentiation marker genes, they did not observe any change in morphology or enrichment of differentiation signature in their gene-expression data. These differences could potentially be attributed to a difference in the length of time the cells were infected with the shRNA before they were examined.

Our data show that JMJD1C modulates the HOXA9-mediated gene-expression program and that HOXA9 physically associates with JMJD1C. The functional importance of this interaction is supported by the fact that the HOXA9-controlled program ranks at the top among differentially expressed gene sets when WT and JMJD1C-deficient cells are compared in both MLL-AF9 and HOXA9/MEIS1 leukemias. Furthermore, we showed enrichment of a set of genes bound and negatively regulated by HOXA9 in murine BM transduced by HOXA9/MEIS1 (refs. 44, 45, and Figure 3B), consistent with findings by Sroczynska et al. (36). Moreover, loss of JMJD1C impairs LSCs in the MLL-AF9 AML model, which is highly dependent on HOXA9 function, as well as in leukemias driven by HOXA9/MEIS1. This has direct relevance for human leukemia, as HOXA9 is highly expressed in approximately $50 \%$ of human AML patient samples $(57,58)$ and has been linked to poor prognosis. In a gene-expression array analysis, $\mathrm{HOXA} 9$ overexpression was most correlated with treatment outcomes (58). Furthermore, HOXA9 has been shown to be required for $M L L$-rearranged leukemia in mouse models $(17,18,20,59)$ and human leukemia cell lines (37), demonstrating an important role in leukemias where it is overexpressed. The data presented here suggest that JMJD1C may have broad importance in human AMLs that overexpress HOXA9.

We have shown that JMJD1C interaction with HOXA9 plays an important role in HOXA9-dependent leukemias, but the precise enzymatic target of JMJD1C remains unclear. JMJD1C has been reported to be a histone $\mathrm{H} 3 \mathrm{~K} 9 \mathrm{me} 1 / 2$ demethylase (40). However, we were unable to detect differences in global H3K9me1/2 levels upon loss of JMJD1C (Supplemental Figure $3 \mathrm{C}$ ), and we were not able to find a correlation between changes in gene expression and H3K9 methylation levels (Supplemental Figure 3D). This is consistent with several recent reports that failed to detect demethylase activity for JMJD1C toward H3K9 methylation when histone $\mathrm{H} 3 \mathrm{~K} 9$-methylated peptides were used as a substrate $(33,36,60)$. However, we cannot rule out the possibility that JMJD1C still has H3K9 demethylase activity because of the lack of direct JMJD1C-binding sites that can be used in the analysis. It also remains possible that JMJD1C has demethylase activity toward other methylated lysines that are critical for the phenotypes we describe. Nonetheless, we assessed global levels of H3K4me3, H3K27me3, and H3K79me2 in Jmjd1 d/f versus $J m j d 1 c^{-1-}$ cells and failed to detect any clear differences (data not shown). Alternatively, JMJD1C may influence HOXA9-mediated gene expression by serving as a scaffold to facilitate recruitment and interaction between HOXA9 and its cofactors or by modulating posttranslational modification of either HOXA9 itself or its binding partners. Along these lines, JMJD1C was recently shown to be a protein demethylase for MDC1, a protein that plays a role in DNA damage repair (61). Future studies will focus on identification of the enzymatic substrates that are demethylated by JMJD1C, as identification of these substrates will likely identify other proteins that play a critical role in LSC biology.

The effect of JMJD1C loss on hematopoiesis appears to be much less profound than that of leukemia. Remarkably, no major defect was observed in steady-state homeostasis after complete loss of JMJD1C. However, loss of JMJD1C does affect competitiveness of HSPCs in serial transplantation, as evidenced by the markedly decreased chimerism in all hematopoietic lineages after competitive transplantation. Moreover, reconstitution of the blood system after 5-Fu administration was also impaired. Our finding that JMJD1C modulates the HOXA9-controlled gene-expression program in leukemia prompted us to examine the possibility of a similar mechanism in normal hematopoiesis. We did not find significant differences in expression of HOXA9-associated programs in the $J m_{j d 1 c^{-1}}$ versus $J m j d 1 c^{+/+}$LSKs. This could be due to the fact that HOXA9 gene sets were all derived from leukemia cells, but not normal hematopoiesis, or that HOXA9 plays a critical role in LSCs, but HSCs have redundant self-renewal mechanisms. Consistently, HOXA9 plays a prominent role in leukemia $(20,37)$. However, the normal hematopoietic phenotype of Hoxa $9^{-/}$mice is similar to that of JMJD1C: mildly decreased hematopoietic progenitor numbers and impaired competitive repopulating activity, but minimal deficiencies of steady-state hematopoiesis $(17,62)$. 
Nonetheless, our data suggest an approach to targeting HOXA9 function in leukemia and perhaps other cancers by targeting an enzyme that is part of the HOXA9 complex. These findings will prompt further study of JMJD1C in leukemia and the development of small-molecule JMJD1C inhibitors.

\section{Methods}

Jmjd1c knockout mouse breeding. Animals were maintained at the Animal Research Facility at Children's Hospital Boston (Boston, Massachusetts, USA) and then at the Research Animal Resource Center at Memorial Sloan Kettering Cancer Center. Jmjd1 $c^{\text {tmla/+ }}$ mice were obtained from EMMA (41). These mice were bred with ACTB-FLPe mice (provided by Stuart Orkin, Division of Pediatric Hematology/ Oncology, Dana Farber Cancer Institute and Boston Children's Hospital, Harvard Medical School, Boston, MA, USA) to generate Jmjd1 $c^{f /+}$ mice. Conditional mice were crossed to Vav1-Cre (provided by Stuart Orkin) or Mx1-Cre mice (The Jackson Laboratory). Detection of WT and floxed alleles of Jmjd1c was performed by PCR using the Jmjd1c 5' arm: CAGAGACAGTCGAACACATTTAGG, the Jmjd1c 3' WT R1: CAACTTAAAACAAATCAACATAAAATAGTAACTTC, and LAR3: CAACGGGTTCTTCTGTTAGTCC with a band size of 283 bp and 549 bp for WT and floxed allele. Detection of floxed and deleted alleles of Jmjd1c was performed by PCR using the Jmjd1c $5^{\prime}$ arm, Jmjd1c-for: TCGAAGCCTAATGGAGTTCTCAGC, and LoxR: TGAACTGATGGCGAGCTCAGACC with a band size of $372 \mathrm{bp}$ (deleted) and $272 \mathrm{bp}$ (floxed).

Mouse experiments. Deletion of floxed alleles in Mx1-Cre mice was induced by i.p. injections with $12.5 \mu \mathrm{g} / \mathrm{g}$ of body weight pIpC (Invivogen) 3 times over a week. For transplantation experiments, mice received either a sublethal ( $6 \mathrm{~Gy}$ ) or a lethal $(9.5 \mathrm{~Gy})$ dose of irradiation prior to tail-vein injection of BM cells. CBC was analyzed by Hemavet 950 (Drew Scientific). For 5-Fu experiments, mice were injected i.p. with $150 \mathrm{mg} / \mathrm{kg}$ of body weight 5 -Fu.

Cell culture. MLL-AF9 transformed preleukemic cells or leukemia was maintained in leukemia media (RPMI-1640 with 10\% FBS and $1 \%$ penicillin/streptomycin [Invitrogen]) or M3234 methylcellulose (Stem Cell Technologies) supplemented with 10 ng/ml IL-3 (Peprotech). Hoxa9/Meis1 and AML-ETO transformed cells were maintained in a manner similar to that of MLL-AF9 cells with $10 \mathrm{ng} / \mathrm{ml}$ of IL- 6 and SCF (Peprotech) in addition to IL-3 in a humidified incubator at $37^{\circ} \mathrm{C}$ in $5 \% \mathrm{CO}_{2}$. Human leukemia cell lines were from ATCC or DSMZ and were cultured in leukemia media as described above. For culturing of the MonoMac6 cell line, $2 \mathrm{mM} \mathrm{L}$-glutamine (Invitrogen), nonessential amino acids (Invitrogen), $1 \mathrm{mM}$ sodium pyruvate (Invitrogen), and 10 $\mu \mathrm{g} / \mathrm{ml}$ human insulin (Sigma-Aldrich) were also added.

Virus production and transduction of cells. The vectors used included pMSCV MLL-AF9-IRES-GFP (14), pMSCV HOXA9-IRES-MEIS1 neo (a gift from Guy Sauvageau, Department of Medicine, Faculty of Medicine, Université de Montréal, Montreal, Quebec, Canada), pMSCV CRE-IRES YFP (a gift from David Williams, Division of Hematology/ Oncology, Boston Children's Hospital), and pMSCV CRE-IRES-Tomato (63). pLKO.1 JMJD1C hairpin constructs were obtained from the Broad Institute RNAi consortium (http://www.broadinstitute.org/rnai/trc): shJMJD1C no. 2: TCCACCTCCAGAGACTATAAA; shJMJD1C no. 3: GAGATGTGGAGACCTAATAAT. Ecotropic retroviruses were generated by contransfection of $293 \mathrm{~T}$ cells with Ecopack packaging vector (Clontech) and respective viral vectors using Fugene 6 (Roche Molecu- lar Biochemicals). Amphotropic lentiviruses were generated similarly using psPAX2 and pMD2 packaging vectors. Viral supernatant collected 48 and 72 hours after transfection was used for transduction of cells. For transduction of LSK cells, $200 \mu$ l of cell suspension (in IMDM media plus $10 \% \mathrm{FBS}, 1 \%$ penicillin/streptomycin [Invitrogen], $10 \mathrm{ng} / \mathrm{ml}$ of IL-3 and IL-6, $40 \mathrm{ng} / \mathrm{ml} \mathrm{SCF}$, and $20 \mathrm{ng} / \mathrm{ml}$ Flt3 ligand and Tpo) and $800 \mu \mathrm{l}$ of viral supernatant were combined in 1 well of a 24 -well plate coated with retronectin (Takara), supplemented with $5 \mu \mathrm{g} / \mathrm{ml}$ Polybrene (American Bioanalytical) and $7.5 \mu \mathrm{l}$ 1M HEPES (Invitrogen). For other transductions, 4 million cells in 2 or $3 \mathrm{ml}$ of appropriate media were combined with 2 or $1 \mathrm{ml}$ of viral supernatant in 1 well of a 6-well plate. Spinfection was conducted in a swing bucket centrifuge at $1,400 \mathrm{~g}$ for 90 minutes at $30^{\circ} \mathrm{C}$. Lentiviral transduction of human cell lines was carried out similarly. To generate transformed preleukemic cells, LSKs transduced with MLL-AF9 or HOXA9/MEIS1 were plated and propagated in M3234 methylcellulose (Stem Cell Technologies) supplemented with cytokines. For generation of leukemia, 1,000 to 3,000 transduced LSKs were transplanted into lethally irradiated $\mathrm{C} 57 \mathrm{BL} / 6$ mice (6-8 weeks) with 0.3 million of helper BM cells.

In vivo shRNA screen. Pooled in vivo shRNA screen was conducted at the Broad Institute RNAi consortium. Pooled lentiviruses for screening were generated by pooling equal amounts of the lentiviral shRNA plasmids prior to lentiviral production (64). Leukemia BM cells were harvested from the moribund tertiary MLL-AF9 mouse and transduced with pooled lentivirus shRNA against MLL-AF9 targets. A total of four 12-well plates were spinfected with $500 \mu$ l of cells (4 million/ml), $100 \mu \mathrm{l}$ ( $30 \%$ infection rate) of pooled viral supernatant, and $5 \mu \mathrm{g} / \mathrm{ml}$ of polybrene per well. Cells were collected the next day ( 4 wells were collected as 1 replicate), and 1.5 million cells in $0.5 \mathrm{ml}$ were injected into sublethally irradiated C57BL/6 recipients. Two weeks later, cells from moribund mice were harvested. shRNA sequences were amplified by PCR from genomic DNA and subjected to Illumina sequencing at the RNAi consortium. Detailed experimental procedures on DNA preparation and sequencing to determine shRNA representation were published previously (65). Raw sequencing data were first normalized to total number of reads for each replicate, and fold change was calculated as ratio of normalized reads between BM or SPL and early time point. Further normalization was done by dividing the ratio above by the ratio of read counts of control shRNAs between the same time points (65).

Flow cytometry and cell sorting. All flow cytometry experiments were performed using a BD FACSAria for sorting and a BD LSRFortessa for analysis. The following antibodies were used: Biotin-Conjugated Mouse Lineage Panel, FITC-CD3 (clone 17A2), FITC-CD45.2 (clone 104) (all from BD); FITC-CD34 (clone RAM34), PE-CD45.2 (clone 104), PE-CD16/32 (clone 93), APC-CD45.1 (clone A20), APC-CD117 (clone 2B8), APC-CD150 (clone TC15-12F12.2), PE-Cy7-Gr19 (clone RB8C5), APC-Cy7 streptavidin, APC-Cy7-B220 (clone RA3-6B2), PB-Sca1 (clone D7), PE-Cy7-CD117 (clone 2B8) (all from BioLegend); FITCCD48 (clone HM48-1), PE-CD127 (clone A7R34), PB-CD11b9 (clone M1/70), APC-CD135 (clone A2F10), PE-Cy7-Sca1 (clone D7), PBCD16/32 (clone 93) (all from eBioscience). To sort LSK cells and HSCs, $\mathrm{BM}$ cells were stained with biotin-conjugated mouse lineage panel (BD Biosciences) and then passed through a MACS lineage depletion column (Miltenyi Biotech). The resulting Lin $^{-}$BM was stained with antibodies against the respective panel of surface markers for $\mathrm{LSK}\left(\mathrm{Lin}^{-} \mathrm{Sca}^{+} \mathrm{C}-\mathrm{Kit}^{+}\right)$ and HSD (CD150 $\left.{ }^{+} \mathrm{CD} 48^{-} \mathrm{LSK}\right)$ and sorted on a FACSAria (BD). 
CFC assay. For myeloid progenitor assay, Cre-Tomato-transduced Jmjd1 $c^{f / f} \mathrm{BM}$ cells were sorted for Tomato cells and plated in $1.5 \mathrm{ml}$ of M3434 (Stem Cell Technologies) in 1 well of a 6-well plate in duplicates. Colony numbers were counted 7 to 10 days later. For leukemic blast CFC assay, Cre-Tomato-transduced WT and Jmjd1 $c^{f / f}$ MLLAF9 leukemic or HOXA9/MEIS1 and AML-ETO preleukemic cells were sorted for GFP/Tomato double-positive cells (Tomato ${ }^{+}$cells for HOXA9/MEIS1) and plated similarly to what is stated above in M3234 supplemented with cytokines.

Proliferation assay, cell cycle, apoptosis assay. For proliferation of WT and Jmjd1c ${ }^{-/-}$MLL-AF9 leukemia cells, $100 \mu \mathrm{l}$ of 10,000/ $\mathrm{ml}$ cells were seeded per well in 96-well plates and counted every 6 days by flow cytometry analysis. Sytox blue (Invitrogen) was used to exclude dead cells.

For cell-cycle analysis of Jmjd1 $c^{-/-}$MLL-AF9 leukemia in vivo, 100 $\mu \mathrm{l}$ of $10 \mathrm{mg} / \mathrm{ml} \mathrm{BrdU}$ (BD Biosciences) was injected into moribund mice by tail vein and, 1 hour later, these mice were sacrificed and BM were harvested. BrdU staining was carried out following the APC BrdU flowing kit (BD Biosciences) protocol and analyzed on a BD LSRFortessa.

Apoptosis assay was performed following instructions for the APC Apoptosis Assay Kit (BD Biosciences) and analyzed on a BD LSRFortessa.

ChIP, ChIP-seq, and RNA-seq. ChIP and ChIP-seq procedures were described previously (28). The antibody used was H3K9me2 (ab1220, Abcam). For RNA-seq, RNA were prepared using TRIzol (Invitrogen), amplified with SMARTer RACE cDNA Amplification Kit (Clontech). Libraries were prepared with the TruSeq RNA Sample Preparation Kit (Illumina) and sequenced on Illumina HiSeq2000.

Cell extracts, IP, and Western blot. Whole-cell SDS lysates were prepared by lysing cells ( 0.2 million) directly in $2 \times$ NuPage LDS sample buffer with sample reducing agent (Invitrogen). Flag-tagged JMJD1C was expressed in the Bac-to-Bac Baculovirus Expression Systems and purified from High Five insect cells as described (Invitrogen). Nuclear extracts were made as described (66) and diluted with BCO (20 mM HEPES, pH 7.9, 0.2 mM EDTA, and 10\% glycerol)/0.2\% Igepal CA-630. IP/co-IP experiments were performed by incubating diluted nuclear extract with strepavidin- (Invitrogen) or HA-conjugated beads overnight. Beads were washed 4 times with BC150 (20 mM HEPES, pH 7.9, 150 $\mathrm{mM} \mathrm{KCl}, 0.2 \mathrm{mM}$ EDTA, and 10\% glycerol) plus 0.1\% Igepal CA-630. Bio-JMJD1C, Bio-KDM3B, and Flag-KDM3A constructs were previously described (60), and N-terminal HA-HOXA9-containing human HOXA9 was cloned into XhoI and EcoRI sites in pMSCV-IRES-GFP. For GST pull-down assay, mouse HOXA9 was amplified from mouse cDNA and cloned into BamHI and EcoRI sites in pGEX-4T1, expressed, and purified as described (GE Life Sciences). GST pull-down was performed under the same conditions as co-IP. Proteins were separated on NuPage Tris-Bis $4 \%$ to $12 \%$ precast gels following the manufacturer's protocol. Gel transfer was performed using iBlot (Invitrogen) to nitrocellulose membrane. The following antibodies were used for immune blotting: H3K9me1 (ab8896, Abcam), H3K9me2 (ab1220, Abcam), H3 (ab1791, Abcam) cleaved caspase-3 (5A1E, Cell Signaling), $\alpha$-HA (ab16918, Abcam), streptavidin HRP (Thermo Scientific), $\alpha$-JMJD1C (17-10262, EMD Millipore), and $\alpha$-HOXA10 (H0916, Sigma-Aldrich).

Data analysis. ChIP-seq data were analyzed as described previously (28). RNA-seq data were analyzed as follows: raw reads were preprocessed using Trim Galore! (http://www.bioinformatics.babraham. ac.uk/projects/trim_galore/) to remove low-quality reads and adapter sequences. Following the QC step, sequence reads were aligned to the mouse genome reference assembly mm 10 (build 10.2) using STAR (67). The number of reads uniquely mapped to each gene (Ensembl 75 annotation) was determined using Htseq (68). To determine the number of genes identified in each sample, we used the union mode in counting reads with Htseq-count. We used DESeq to identify the differentially expressed genes (69). The $P$ values were adjusted for multiple testing by the Benjamini-Hochberg method. The RNA-seq pipeline from Basepair (www.basepair.io) was also used in the analysis. Expression count was analyzed by STAR and differential expression by DESeq. The integrative analysis of histone modification and gene expression was done using iCanPlot (70). All RNA-seq and ChIP-seq data sets were deposited in the NCBI's Gene Expression Omnibus (GEO GSE75581). LSC frequency was calculated using L-Calc software (Stemcell Technologies).

Statistics. Error bars in all graphs represents mean \pm SEM. Statistical analysis of means between 2 populations was performed using 2-tailed, unpaired Student's $t$ test. Survival curves were assessed by Log-rank test. An approximate $P$ value for nonparametric correlation was calculated for the Pearson correlation. A $P$ value for overlap between gene sets in the Venn diagram was calculated by exact hypergeometric probability. A $P$ value of less than 0.05 was considered significant.

Study approval. All in vivo experiments in animals were approved by the Memorial Sloan Cancer Center and Medical College of Wisconsin Animal Care and Use Committees.

\section{Author contributions}

NZ and SAA conceived the study and wrote the paper. NFR, AUS, and RK analyzed Illumina sequencing data. FAS and BLE provided help with the shRNA screen and analyzed the screen result. MC and RGR performed and helped with the protein-protein interaction study and provided conceptual input. NZ, RE, and JD performed experiments with help from JCM, CWC, and AJD. HX and SHC contributed to study design and data analysis.

\section{Acknowledgments}

We want to thank John Doench for assistance with RNAi screening. S.A. Armstrong was supported by grants from the American Cancer Society, the Leukemia and Lymphoma Society, the Gabrielle's Angel Foundation, and the National Cancer Institute (U01CA105423, R01CA140575, and P01CA66996). N. Zhu was supported by a grant from the National Cancer Institute (K99/R00 CA168996). Support was also provided by NIH grant P30CA008748 to Memorial Sloan Kettering Cancer Center.

Address correspondence to: Scott A. Armstrong, Memorial Sloan Kettering Cancer Center, 1275 York Avenue, New York, New York 10065, USA. Phone: 646.888.3479; E-mail: armstros@mskcc.org.

Rowena Eng and Janna C. Minehart's current address: New York University School of Medicine, New York, New York, USA.

Fatima Al-Shahrour's current address: Spanish National Cancer Research Centre, Madrid, Spain.

Aniruddha J. Deshpande's current address: Sanford-Burnham Medical Research Institute, La Jolla, California, USA. 
1. Reya T, Morrison SJ, Clarke MF, Weissman IL. Stem cells, cancer, and cancer stem cells. Nature. 2001;414(6859):105-111.

2 . Wang $\mathrm{Y}$, et al. The Wnt $/ \beta$-catenin pathway is required for the development of leukemia stem cells in AML. Science. 2010;327(5973):1650-1653.

3. Koch U, Wilson A, Cobas M, Kemler R, Macdonald HR, Radtke F. Simultaneous loss of $\beta$ - and $\gamma$-catenin does not perturb hematopoiesis or lymphopoiesis. Blood. 2008;111(1):160-164.

4. Dierks C, et al. Expansion of Bcr-Abl-positive leukemic stem cells is dependent on Hedgehog pathway activation. Cancer Cell. 2008;14(3):238-249.

5. Zhao C, et al. Hedgehog signalling is essential for maintenance of cancer stem cells in myeloid leukaemia. Nature. 2009;458(7239):776-779.

6. Hofmann I, et al. Hedgehog signaling is dispensable for adult murine hematopoietic stem cell function and hematopoiesis. Cell Stem Cell. 2009;4(6):559-567.

7. Nakamura T, et al. ALL-1 is a histone methyltransferase that assembles a supercomplex of proteins involved in transcriptional regulation. Mol Cell. 2002;10(5):1119-1128.

8. Milne TA, et al. MLL targets SET domain methyltransferase activity to Hox gene promoters. Mol Cell. 2002;10(5):1107-1117.

9. Yu BD, Hess JL, Horning SE, Brown GA, Korsmeyer SJ. Altered Hox expression and segmental identity in Mll-mutant mice. Nature. 1995;378(6556):505-508.

10. Yagi H, Deguchi K, Aono A, Tani Y, Kishimoto $\mathrm{T}$, Komori T. Growth disturbance in fetal liver hematopoiesis of Mll-mutant mice. Blood. 1998;92(1):108-117.

11. Ernst P, Mabon M, Davidson AJ, Zon LI, Korsmeyer SJ. An Mll-dependent Hox program drives hematopoietic progenitor expansion. Curr Biol. 2004;14(22):2063-2069.

12. Jude CD, Climer L, Xu D, Artinger E, Fisher JK, Ernst P. Unique and independent roles for MLL in adult hematopoietic stem cells and progenitors. Cell Stem Cell. 2007;1(3):324-337.

13. Krivtsov AV, Armstrong SA. MLL translocations, histone modifications and leukaemia stem-cell development. Nat Rev Cancer. 2007;7(11):823-833.

14. Krivtsov AV, et al. Transformation from committed progenitor to leukaemia stem cell initiated by MLL-AF9. Nature. 2006;442(7104):818-822.

15. Somervaille TC, Cleary ML. Identification and characterization of leukemia stem cells in murine MLL-AF9 acute myeloid leukemia. Cancer Cell. 2006;10(4):257-268.

16. Armstrong SA, et al. MLL translocations specify a distinct gene expression profile that distinguishes a unique leukemia. Nat Genet. 2002;30(1):41-47.

17. So CW, Karsunky H, Wong P, Weissman IL, Cleary ML. Leukemic transformation of hematopoietic progenitors by MLL-GAS7 in the absence of Hoxa7 or Hoxa9. Blood. 2004;103(8):3192-3199.

18. Zeisig BB, et al. Hoxa9 and Meis1 are key targets for MLL-ENL-mediated cellular immortalization. Mol Cell Biol. 2004;24(2):617-628.

19. Kumar AR, Hudson WA, Chen W, Nishiuchi R, Yao Q, Kersey JH. Hoxa9 influences the phenotype but not the incidence of Mll-AF9 fusion gene leukemia. Blood. 2004;103(5):1823-1828.
20. Ayton PM, Cleary ML. Transformation of myeloid progenitors by MLL oncoproteins is dependent on Hoxa7 and Hoxa9. Genes Dev. 2003;17(18):2298-2307.

21. Krivtsov AV, Feng Z, Armstrong SA. Transformation from committed progenitor to leukemia stem cells. Ann N Y Acad Sci. 2009;1176:144-149.

22. Zuber J, et al. An integrated approach to dissecting oncogene addiction implicates a Myb-coordinated self-renewal program as essential for leukemia maintenance. Genes Dev. 2011;25(15):1628-1640.

23. Park IK, et al. Bmi-1 is required for maintenance of adult self-renewing haematopoietic stem cells. Nature. 2003;423(6937):302-305.

24. Lessard J, Sauvageau G. Bmi-1 determines the proliferative capacity of normal and leukaemic stem cells. Nature. 2003;423(6937):255-260.

25. Neff T, et al. Polycomb repressive complex 2 is required for MLL-AF9 leukemia. Proc Natl Acad Sci U S A. 2012;109(13):5028-5033.

26. Xie H, et al. Polycomb repressive complex 2 regulates normal hematopoietic stem cell function in a developmental-stage-specific manner. Cell Stem Cell. 2014;14(1):68-80.

27. Nguyen AT, He J, Taranova O, Zhang Y. Essential role of DOT1L in maintaining normal adult hematopoiesis. Cell Res. 2011;21(9):1370-1373.

28. Bernt KM, et al. MLL-rearranged leukemia is dependent on aberrant H3K79 methylation by DOT1L. Cancer Cell. 2011;20(1):66-78.

29. Jo SY, Granowicz EM, Maillard I, Thomas D, Hess JL. Requirement for Dot1l in murine postnatal hematopoiesis and leukemogenesis by MLL translocation. Blood. 2011;117(18):4759-4768.

30. Tan J, et al. CBX8, a polycomb group protein, is essential for MLL-AF9-induced leukemogenesis. Cancer Cell. 2011;20(5):563-575.

31. Lee JW, Choi HS, Gyuris J, Brent R, Moore DD. Two classes of proteins dependent on either the presence or absence of thyroid hormone for interaction with the thyroid hormone receptor. Mol Endocrinol. 1995;9(2):243-254.

32. Wolf SS, Patchev VK, Obendorf M. A novel variant of the putative demethylase gene, s-JMJD1C, is a coactivator of the AR. Arch Biochem Biophys. 2007;460(1):56-66.

33. Kuroki S, et al. JMJD1C, a JmjC domaincontaining protein, is required for long-term maintenance of male germ cells in mice. Biol Reprod. 2013;89(4):93.

34. Wang J, Park JW, Drissi H, Wang X, Xu RH. Epigenetic regulation of miR-302 by JMJD1C inhibits neural differentiation of human embryonic stem cells. J Biol Chem. 2014;289(4):2384-2395.

35. Wang L, et al. Novel somatic and germline mutations in intracranial germ cell tumours. Nature. 2014;511(7508):241-245.

36. Sroczynska P, et al. shRNA screening identifies JMJD1C as being required for leukemia maintenance. Blood. 2014;123(12):1870-1882.

37. Faber J, et al. HOXA9 is required for survival in human MLL-rearranged acute leukemias. Blood. 2009;113(11):2375-2385.

38. Wong P, Iwasaki M, Somervaille TC, So CW, Cleary ML. Meis1 is an essential and rate-limiting regulator of MLL leukemia stem cell potential. Genes Dev. 2007;21(21):2762-2774
39. Ross ME, et al. Gene expression profiling of pediatric acute myelogenous leukemia. Blood. 2004;104(12):3679-3687.

40. Kim SM, et al. Regulation of mouse steroidogenesis by WHISTLE and JMJD1C through histone methylation balance. Nucleic Acids Res. 2010;38(19):6389-6403.

41. Skarnes WC, et al. A conditional knockout resource for the genome-wide study of mouse gene function. Nature. 2011;474(7351):337-342.

42. Subramanian A, et al. Gene set enrichment analysis: a knowledge-based approach for interpreting genome-wide expression profiles. Proc Natl Acad Sci U S A. 2005;102(43):15545-15550.

43. Mootha VK, et al. PGC-1 $\alpha$-responsive genes involved in oxidative phosphorylation are coordinately downregulated in human diabetes. Nat Genet. 2003;34(3):267-273.

44. Huang $Y$, et al. Identification and characterization of Hoxa9 binding sites in hematopoietic cells. Blood. 2012;119(2):388-398.

45. Lehnertz B, et al. The methyltransferase G9a regulates HoxA9-dependent transcription in AML. Genes Dev. 2014;28(4):317-327.

46. Argiropoulos B, Humphries RK. Hox genes in hematopoiesis and leukemogenesis. Oncogene. 2007;26(47):6766-6776.

47. Kroon E, Krosl J, Thorsteinsdottir U, Baban S, Buchberg AM, Sauvageau G. Hoxa9 transforms primary bone marrow cells through specific collaboration with Meis1a but not Pbx1b. EMBO J. 1998;17(13):3714-3725.

48. Yan M, et al. A previously unidentified alternatively spliced isoform of $\mathrm{t}(8 ; 21)$ transcript promotes leukemogenesis. Nat Med. 2006;12(8):945-949.

49. Georgiades P, et al. VavCre transgenic mice: a tool for mutagenesis in hematopoietic and endothelial lineages. Genesis. 2002;34(4):251-256.

50. Orford KW, Scadden DT. Deconstructing stem cell self-renewal: genetic insights into cell-cycle regulation. Nat Rev Genet. 2008;9(2):115-128.

51. Pietras EM, Warr MR, Passegue E. Cell cycle regulation in hematopoietic stem cells. JCell Biol. 2011;195(5):709-720.

52. Zhao M, et al. Megakaryocytes maintain homeostatic quiescence and promote post-injury regeneration of hematopoietic stem cells. Nat Med. 2014;20(11):1321-1326.

53. Dawson MA, Kouzarides T. Cancer epigenetics: from mechanism to therapy. Cell. 2012;150(1):12-27.

54. Baylin SB, Jones PA. A decade of exploring the cancer epigenome - biological and translational implications. Nat Rev Cancer. 2011;11(10):726-734.

55. Shih AH, Abdel-Wahab O, Patel JP, Levine RL. The role of mutations in epigenetic regulators in myeloid malignancies. Nat Rev Cancer. 2012;12(9):599-612.

56. Neff T, Armstrong SA. Recent progress toward epigenetic therapies: the example of mixed lineage leukemia. Blood. 2013;121(24):4847-4853.

57. Andreeff M, et al. HOX expression patterns identify a common signature for favorable AML. Leukemia. 2008;22(11):2041-2047.

58. Golub TR, et al. Molecular classification of cancer: class discovery and class prediction by gene expression monitoring. Science. 
1999;286(5439):531-537.

59. Horton SJ, et al. Continuous MLL-ENL expression is necessary to establish a "Hox Code" and maintain immortalization of hematopoietic progenitor cells. Cancer Res. 2005;65(20):9245-9252.

60. Brauchle M, et al. Protein complex interactor analysis and differential activity of KDM3 subfamily members towards H3K9 methylation. PLoS One. 2013;8(4):e60549.

61. Watanabe S, et al. JMJD1C demethylates MDC1 to regulate the RNF8 and BRCA1-mediated chromatin response to DNA breaks. Nat Struct Mol Biol. 2013;20(12):1425-1433.

62. Lawrence HJ, et al. Loss of expression of the Hoxa-9 homeobox gene impairs the proliferation and repopulating ability of hematopoietic stem cells. Blood. 2005;106(12):3988-3994.

63. Chen L, et al. Abrogation of MLL-AF10 and CALM-AF10-mediated transformation through genetic inactivation or pharmacological inhibition of the H3K79 methyltransferase Dot1l. Leukemia. 2013;27(4):813-822.

64. Luo B, et al. Highly parallel identification of essential genes in cancer cells. Proc Natl Acad Sci U S A. 2008;105(51):20380-20385.

65. Miller PG, et al. In vivo RNAi screening identifies a leukemia-specific dependence on integrin $\beta 3$ signaling. Cancer Cell. 2013;24(1):45-58.

66. Dignam JD, Lebovitz RM, Roeder RG. Accurate transcription initiation by RNA polymerase II in a soluble extract from isolated mammalian nuclei. Nucleic Acids Res. 1983;11(5):1475-1489.

67. Dobin A, et al. STAR: ultrafast universal RNA-seq aligner. Bioinformatics. 2013;29(1):15-21.

68. Anders S, et al. Count-based differential expression analysis of RNA sequencing data using R and Bioconductor. Nat Protoc. 2013;8(9):1765-1786.

69. Anders S, Huber W. Differential expression analysis for sequence count data. Genome Biol. 2010;11(10):R106.

70. Sinha AU, Armstrong SA. iCanPlot: visual exploration of high-throughput omics data using interactive Canvas plotting. PLoS One. 2012;7(2):e31690. 